\title{
Rationality of some tetragonal loci
}

\author{
Shouhei Ma
}

\begin{abstract}
We prove that the moduli space of tetragonal curves of genus $g \geqslant 7$ is rational when $g \equiv 1,2,5,6,9,10$ modulo 12 and $g \neq 9,45$.
\end{abstract}

\section{Introduction}

Let $\mathcal{M}_{g}$ be the moduli space of curves of genus $g \geqslant 7$, and let $\mathcal{T}_{g} \subset \mathcal{M}_{g}$ be the locus of tetragonal curves, namely non-hyperelliptic curves which have a map of degree four to $\mathbb{P}^{1}$. Classically, $\mathcal{T}_{g}$ has been known to be unirational ([Pet23], [AC81], [Sch86]), but the question whether it is rational remained open until recently, when Böhning, Bothmer and Casnati [BvBC12] proved that $\mathcal{T}_{7}$ is rational. In this article we make a further step in this direction, showing that $\mathcal{T}_{g}$ is rational for about half of the genera.

TheOREM 1.1. Let $g \geqslant 7$ be a natural number with

$$
g \equiv 1,2,5,6,9,10 \bmod 12
$$

and $g \neq 9,45$. Then the tetragonal locus $\mathcal{T}_{g}$ is rational.

This extends the series of rationality results for the hyperelliptic loci ([Kat84a], [BK85]) and the trigonal loci ([She85], [Ma13a], [Ma12]). There naturally arises the question at which gonality we no longer can expect rationality. One might approach pentagonal loci as well using the description in [Sch86], but it seems that only little is known for gonality $\geqslant 6$ ([Gei12]).

One basic approach for proving rationality of a moduli space is to first describe it birationally as the quotient of a parameter space $U$ by an algebraic group $G$, and then analyze the $G$-action on $U$. The first step means giving a construction of general members that is canonical. In the present case, we use Schreyer's model [Sch86], which describes a tetragonal curve $C$ as a complete intersection of two relative conics in a $\mathbb{P}^{2}$-bundle over $\mathbb{P}^{1}$. When $C$ is general, the ambient $\mathbb{P}^{2}$ bundle $X$ is either (i) $\mathbb{P}^{1} \times \mathbb{P}^{2}$, (ii) the blow-up of $\mathbb{P}^{3}$ along a line or (iii) a small resolution of a quadric cone in $\mathbb{P}^{4}$, depending on $[g] \in \mathbb{Z} / 3 \mathbb{Z}$. Thus, in the present case, $U$ is a parameter space of some complete intersection curves in that $X$, and $G$ is the automorphism group of $X$. The structure of $U$ varies according to the parity of $g$, so the nature of the group action that we will study primarily depends on $[g] \in \mathbb{Z} / 6 \mathbb{Z}$. Moreover, when attacking the rationality problem, we were faced with a technical obstruction which caused the further mod 12 condition in Theorem 1.1 .

Received 2 August 2013, accepted in final form 10 February 2014.

2010 Mathematics Subject Classification 14H45 (primary), 14H10, $14 \mathrm{E} 08$ (secondary).

Keywords: tetragonal curve, moduli space, rationality

This journal is (C) Foundation Compositio Mathematica 2014. This article is distributed with Open Access under the terms of the Creative Commons Attribution Non-Commercial License, which permits non-commercial reuse, distribution, and reproduction in any medium, provided that the original work is properly cited. For commercial re-use, please contact the Foundation Compositio Mathematica.

Supported by Grant-in-Aid for Scientific Research No.12809324 and No.22224001. 


\section{SHOUHEI MA}

We work over the complex numbers. Section 2 contains preliminaries on the relevant $\mathbb{P}^{2}$ bundles. In $\S 3$ we derive a birational description of $\mathcal{T}_{g}$ as a quotient space. Section 4 is a collection of miscellaneous techniques for proving the rationality of quotient spaces. They will be also useful for other rationality problems. Theorem 1.1 is proved in $\S 5-\S 7$ : this division comes from the above classification (i) - (iii).

\section{Notation}

We will use the following notation for irreducible representations of $\mathrm{SL}_{2}$ and $\mathrm{SL}_{2} \times \mathrm{SL}_{2}$ :

$$
\begin{gathered}
V_{d}=H^{0}\left(\mathcal{O}_{\mathbb{P}^{1}}(d)\right), \\
V_{d, e}=V_{d} \otimes V_{e}=H^{0}\left(\mathcal{O}_{\mathbb{P}^{1} \times \mathbb{P}^{1}}(d, e)\right) .
\end{gathered}
$$

The space $V_{d}$ is also regarded as a $\mathrm{GL}_{2}$-representation in the natural way.

\section{Three-dimensional scrolls}

For two natural numbers $0 \leqslant e \leqslant f$, let $\mathcal{E}_{e, f}$ be the vector bundle

$$
\mathcal{O}_{\mathbb{P}^{1}} \oplus \mathcal{O}_{\mathbb{P}^{1}}(-e) \oplus \mathcal{O}_{\mathbb{P}^{1}}(-f)
$$

over $\mathbb{P}^{1}$, and let

$$
X_{e, f}=\mathbb{P} \mathcal{E}_{e, f}
$$

be the associated $\mathbb{P}^{2}$-bundle parametrizing lines in the fibers of $\mathcal{E}_{e, f}$. We denote by $\pi: X_{e, f} \rightarrow \mathbb{P}^{1}$ the natural projection. In the convention of Grothendieck, $X_{e, f}$ is rather the projectivization of the dual $\mathcal{E}_{e, f}^{\vee}$. Thus $\pi_{*} \mathcal{O}_{\pi}(1) \simeq \mathcal{E}_{e, f}^{\vee}$ for the relative hyperplane bundle $\mathcal{O}_{\pi}(1)$. These $\mathbb{P}^{2}$-bundles play a fundamental role in the study of tetragonal curves. In $\S 2.1$ we recall their basic properties following Schreyer [Sch86]. When studying birational types of tetragonal loci, we actually use only three $\mathbb{P}^{2}$-bundles: $X_{0,0}=\mathbb{P}^{1} \times \mathbb{P}^{2}, X_{0,1}$ and $X_{1,1}$. In $\S 2.2$ and $\S 2.3$, we take a closer look at $X_{0,1}$ and $X_{1,1}$.

\subsection{Basic properties}

The Picard group of $X_{e, f}$ is freely generated by $\mathcal{O}_{\pi}(1)$ and $\pi^{*} \mathcal{O}_{\mathbb{P}^{1}}(1)$. Accordingly, we will write

$$
L_{a, b}=\mathcal{O}_{\pi}(a) \otimes \pi^{*} \mathcal{O}_{\mathbb{P}^{1}}(b) .
$$

For example, the canonical bundle of $X_{e, f}$ is isomorphic to $L_{-3,-2+e+f}$. This can be seen by considering the relative Euler sequence

$$
0 \rightarrow \mathcal{O}_{X_{e, f}} \rightarrow \pi^{*} \mathcal{E}_{e, f} \otimes \mathcal{O}_{\pi}(1) \rightarrow T_{\pi} \rightarrow 0,
$$

where $T_{\pi}$ is the relative tangent bundle. The intersection numbers between line bundles are calculated from

$$
\left(L_{1,0} \cdot L_{1,0} \cdot L_{1,0}\right)=e+f, \quad\left(L_{1,0} \cdot L_{1,0} \cdot L_{0,1}\right)=1, \quad L_{0,1} \cdot L_{0,1} \equiv 0 .
$$

When $a \geqslant 0$ and $b \geqslant-1$, using $\pi_{*} L_{a, b} \simeq \operatorname{Sym}^{a} \mathcal{E}_{e, f}^{\vee} \otimes \mathcal{O}_{\mathbb{P} 1}(b)$, we have

$$
h^{0}\left(L_{a, b}\right)=(e+f)\left(\begin{array}{c}
a+2 \\
3
\end{array}\right)+(b+1)\left(\begin{array}{c}
a+2 \\
2
\end{array}\right)
$$

$([\mathrm{Sch} 86])$ and $h^{i}\left(L_{a, b}\right)=0$ for $i>0$. 


\section{RATIONALITY OF SOME TETRAGONAL LOCI}

If $b \geqslant 0$ with $(e, f, b) \neq(0,0,0)$, the bundle $L_{1, b}$ is base-point-free and the morphism

$$
\phi=\phi_{L_{1, b}}: X_{e, f} \rightarrow\left|L_{1, b}\right|^{\vee} \simeq \mathbb{P}^{N}, \quad N=e+f+3 b+2,
$$

is birational onto its image. It is an embedding if $b>0$. The $\pi$-fibers are mapped by $\phi$ isomorphically to planes in $\left|L_{1, b}\right|^{\vee}$, which sweep out $\phi\left(X_{e, f}\right)$. The projective variety $\phi\left(X_{e, f}\right)$ is usually called a three-dimensional rational normal scroll. Its scroll type ([Sch86]) is $(b+f, b+e, b)$.

We will be concerned with the automorphism group of $X_{e, f}$. By the relation (2.1), any automorphism acts on $\operatorname{Pic}\left(X_{e, f}\right)$ trivially and in particular preserves $\pi$. Hence we have the basic exact sequence

$$
1 \rightarrow \operatorname{Aut}\left(\mathcal{E}_{e, f}\right) / \mathbb{C}^{\times} \rightarrow \operatorname{Aut}\left(X_{e, f}\right) \rightarrow \mathrm{PGL}_{2} \rightarrow 1
$$

where $\operatorname{Aut}\left(\mathcal{E}_{e, f}\right)$ is the group of bundle automorphisms which are the identity over the base. In this article we refrain from working with $\operatorname{Aut}\left(X_{e, f}\right)$ for general $(e, f)$ and restrict ourselves to $X_{0,0}, X_{0,1}$ and $X_{1,1}$, giving an ad hoc treatment. Note that $\operatorname{Aut}\left(X_{0,0}\right)$ is just $\mathrm{PGL}_{2} \times \mathrm{PGL}_{3}$. The other two cases will be studied in $\S 2.2$ and $\S 2.3$. Here we just mention the following general duality.

Lemma 2.1. We have an isomorphism $\operatorname{Aut}\left(X_{e, f}\right) \simeq \operatorname{Aut}\left(X_{f-e, f}\right)$ of algebraic groups.

Proof. It is convenient to consider the double cover

$$
\tilde{G}=\mathrm{SL}_{2} \ltimes\left(\operatorname{Aut}\left(\mathcal{E}_{e, f}\right) / \mathbb{C}^{\times}\right)
$$

of $\operatorname{Aut}\left(X_{e, f}\right)$, where $\mathrm{SL}_{2}$ acts on $X_{e, f}$ and $\operatorname{Aut}\left(\mathcal{E}_{e, f}\right)$ through the $\mathrm{SL}_{2}$-linearization of $\mathcal{E}_{e, f}$. The kernel of the natural covering map $\tilde{G} \rightarrow \operatorname{Aut}\left(X_{e, f}\right)$ is generated by $\left(-1,(-1)^{*}\right)$. On the other hand, by the canonical isomorphism $\operatorname{Aut}\left(\mathcal{E}_{e, f}\right) \simeq \operatorname{Aut}\left(\mathcal{E}_{e, f}^{\vee}\right)$ and by the $\mathrm{SL}_{2}$-linearization of $\mathcal{E}_{e, f}^{\vee}$, we have a surjective homomorphism $\tilde{G} \rightarrow \operatorname{Aut}\left(\mathbb{P}_{e, f}^{\vee}\right)$. Its kernel is also generated by $\left(-1,(-1)^{*}\right)$. Hence we have $\operatorname{Aut}\left(X_{e, f}\right) \simeq \operatorname{Aut}\left(\mathbb{P} \mathcal{E}_{e, f}^{\vee}\right)$. Finally, $\mathbb{P} \mathcal{E}_{e, f}^{\vee}$ is canonically isomorphic to $X_{f-e, f}$.

\subsection{The bundle $X_{0,1}$ as a blown-up $\mathbb{P}^{3}$}

The $\mathbb{P}^{2}$-bundle $X_{0,1}=\mathbb{P} \mathcal{E}_{0,1}$, where $\mathcal{E}_{0,1}=\mathcal{O}_{\mathbb{P}^{1}}^{\oplus 2} \oplus \mathcal{O}_{\mathbb{P}^{1}}(-1)$, contains the special surface $\Sigma=$ $\mathbb{P O}_{\mathbb{P} 1}^{\oplus 2}$ which is invariant under $\operatorname{Aut}\left(X_{0,1}\right)$. Since a section of $\mathcal{O}_{\mathbb{P} 1}(1) \subset \mathcal{E}_{0,1}^{\vee}$ defines the divisor $\Sigma+F \in\left|\mathcal{O}_{\pi}(1)\right|$, where $F$ is a $\pi$-fiber, $\Sigma$ is (the unique) member of $\left|L_{1,-1}\right|$. We shall distinguish the two rulings on $\Sigma \simeq \mathbb{P}^{1} \times \mathbb{P}^{1}$ by letting $\left.\pi\right|_{\Sigma}: \Sigma \rightarrow \mathbb{P}^{1}$ be the first projection, and the other ruling be the second. In particular, $\left.L_{0,1}\right|_{\Sigma} \simeq \mathcal{O}_{\Sigma}(1,0)$. By the adjunction formula we see that $\left.L_{1,0}\right|_{\Sigma} \simeq \mathcal{O}_{\Sigma}(0,1)$.

\section{LEMma 2.2. The morphism}

$$
\phi=\phi_{\mathcal{O}_{\pi}(1)}: X_{0,1} \rightarrow\left|\mathcal{O}_{\pi}(1)\right|^{\vee} \simeq \mathbb{P}^{3}
$$

is the blow-up along a line $l \subset \mathbb{P}^{3}$ with exceptional divisor $\Sigma$, and $\pi: X_{0,1} \rightarrow \mathbb{P}^{1}$ is obtained as the resolution of the projection $\mathbb{P}^{3} \rightarrow \mathbb{P}^{1}$ from $l$.

Proof. We see that $\phi$ is birational because $\left(\mathcal{O}_{\pi}(1)\right)^{3}=1$. Since $\left|\mathcal{O}_{\pi}(1) \|_{\Sigma}=\right| \mathcal{O}_{\Sigma}(0,1) \mid$, the morphism $\phi$ maps $\Sigma$ to a line $l$, contracting the second ruling and mapping the fibers of the first ruling isomorphically to $l$. On the other hand, each $\pi$-fiber is mapped isomorphically to a plane containing $l$. This implies our claim.

Since $L_{a, b} \simeq \phi^{*} \mathcal{O}_{\mathbb{P}^{3}}(a+b) \otimes \mathcal{O}_{X_{0,1}}(-b \Sigma)$, we can identify $\left|L_{a, b}\right|$ with the linear system of surfaces of degree $a+b$ in $\mathbb{P}^{3}$ which have multiplicity $\geqslant b$ along $l$. To describe it explicitly, take 


\section{Shouhei MA}

homogeneous coordinates $\left[X_{0}, \cdots, X_{3}\right]$ of $\mathbb{P}^{3}$ and let $l$ be defined by $X_{0}=X_{1}=0$. Then the subspace $H^{0}\left(L_{a, b}\right) \subset H^{0}\left(\mathcal{O}_{\mathbb{P}^{3}}(a+b)\right)$ is given by

$$
\bigoplus_{i=b}^{a+b} V_{i}\left(X_{0}, X_{1}\right) \otimes V_{a+b-i}\left(X_{2}, X_{3}\right)
$$

where $V_{d}\left(X_{s}, X_{t}\right)$ denotes the space of homogeneous polynomials of degree $d$ in variables $X_{s}, X_{t}$.

We can regard $\operatorname{Aut}\left(X_{0,1}\right)$ as the subgroup of $\mathrm{PGL}_{4}$ stabilizing $l$. It is convenient to consider inside $\mathrm{GL}_{4}$ the following double cover of $\operatorname{Aut}\left(X_{0,1}\right)$ :

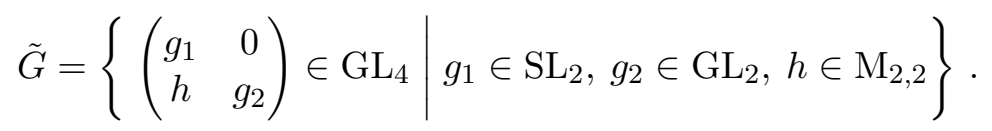

This group is naturally isomorphic to the semidirect product

$$
\tilde{G} \simeq\left(\mathrm{SL}_{2} \times \mathrm{GL}_{2}\right) \ltimes \operatorname{Hom}\left(V_{1}^{(1)}, V_{1}^{(2)}\right),
$$

where $V_{1}^{(1)}=\mathbb{C}\left\langle X_{0}, X_{1}\right\rangle$ and $V_{1}^{(2)}=\mathbb{C}\left\langle X_{2}, X_{3}\right\rangle$ are two copies of $V_{1}, \mathrm{GL}_{2}$ acts on $V_{1}^{(2)}$ in the standard way, and $\mathrm{SL}_{2}$ acts on $\left(V_{1}^{(1)}\right)^{\vee}$ by the dual representation of its standard action on $V_{1}^{(1)}$. The kernel of the projection $\tilde{G} \rightarrow \operatorname{Aut}\left(X_{0,1}\right)$ is generated by $(-1,-1) \in \mathrm{SL}_{2} \times \mathrm{GL}_{2}$.

Now $H^{0}\left(L_{a, b}\right)$ is a $\tilde{G}$-representation, and (2.3) gives its irreducible decomposition under the subgroup $\mathrm{SL}_{2} \times \mathrm{GL}_{2} \subset \tilde{G}$ : the $i$ th summand in $(2.3)$ is the $\mathrm{SL}_{2} \times \mathrm{GL}_{2}$-representation $V_{i, a+b-i}$. To express the action of the unipotent radical $\operatorname{Hom}\left(V_{1}, V_{1}\right)$, for $h \in \operatorname{Hom}\left(V_{1}, V_{1}\right)$ we set

$$
\exp (h)=\left(1, h, h^{\otimes 2} / 2, \cdots\right) \in \underset{d \geqslant 0}{\oplus} \operatorname{Hom}\left(V_{1}, V_{1}\right)^{\otimes d} .
$$

Then $h$ acts on $H^{0}\left(L_{a, b}\right)$ by the linear maps

$$
\langle\cdot, \exp (h)\rangle: V_{i, a+b-i} \rightarrow \underset{d \geqslant 0}{\oplus} V_{i+d, a+b-i-d}
$$

induced from the multiplication $V_{i} \times V_{1} \rightarrow V_{i+1}$ and the contraction $V_{j} \times V_{1}^{\vee} \rightarrow V_{j-1}$. In particular, the subspace $F_{i}=\oplus_{j \geqslant i} V_{j, a+b-j}$ is $\tilde{G}$-invariant. It is the space of polynomials of degree $a+b$ vanishing to order $\geqslant i$ along $l$, that is, $F_{i} \simeq H^{0}\left(L_{a+b-i, i}\right)$. Geometrically the quotient map $H^{0}\left(L_{a, b}\right) \rightarrow H^{0}\left(L_{a, b}\right) / F_{i}$ gives the Taylor expansions up to $(i-b-1)$ th order of the sections of $L_{a, b}$ along $\Sigma$. Here note that $\left.L_{a, b}\right|_{\Sigma} \otimes\left(N_{\Sigma / X_{0,1}}\right)^{-k} \simeq \mathcal{O}_{\Sigma}(b+k, a-k)$.

We remark that $L_{a, b}$ admits a $\tilde{G}$-linearization through that of $\mathcal{O}_{\mathbb{P} 3}(a+b)$ and the ideal sheaf $\mathcal{I}_{l}^{b}$ (for $b \geqslant 0$ ). Since the element $(-1,-1) \in \mathrm{SL}_{2} \times \mathrm{GL}_{2}$ acts on $L_{a, b}$ by multiplication by $(-1)^{a+b}$, we have the following result.

Lemma 2.3. The bundle $L_{a, b}$ is $\operatorname{Aut}\left(X_{0,1}\right)$-linearized when $a+b$ is even.

\subsection{The bundle $X_{1,1}$ as a small resolution of a quadric cone}

The $\mathbb{P}^{2}$-bundle $X_{1,1}=\mathbb{P} \mathcal{E}_{1,1}$, where $\mathcal{E}_{1,1}=\mathcal{O}_{\mathbb{P} 1} \oplus \mathcal{O}_{\mathbb{P} 1}(-1)^{\oplus 2}$, admits the special section $\sigma=\mathbb{P} \mathcal{O}_{\mathbb{P} 1}$ which is invariant under $\operatorname{Aut}\left(X_{1,1}\right)$. Since $\left(\mathcal{O}_{\pi}(1) \cdot \sigma\right)=0$, the morphism

$$
\phi=\phi_{\mathcal{O}_{\pi}(1)}: X_{1,1} \rightarrow\left|\mathcal{O}_{\pi}(1)\right|^{\vee} \simeq \mathbb{P}^{4}
$$

contracts $\sigma$ to a point, say $p_{0}$.

LEMma 2.4. The image $Q=\phi\left(X_{1,1}\right)$ is the quadric cone over a smooth quadric $Q_{0} \subset \mathbb{P}^{3}$ with vertex $p_{0}$, and $\phi: X_{1,1} \rightarrow Q$ is a small resolution of $p_{0}$ with exceptional curve $\sigma$. 


\section{RATIONALITY OF SOME TETRAGONAL LOCI}

Proof. Since $\left(\mathcal{O}_{\pi}(1)\right)^{3}=2$ and $Q$ is nondegenerate, $Q$ must be a quadric hypersurface and $\phi: X_{1,1} \rightarrow Q$ is birational. The $\pi$-fibers are mapped isomorphically to planes, which intersect each other at $p_{0}$. Swept out by those planes, $Q$ must be a quadric cone with vertex $p_{0}$.

Let $f: Q \rightarrow Q_{0}$ be the projection from $p_{0}$. Via the pullback by $f$, the two rulings on $Q_{0} \simeq$ $\mathbb{P}^{1} \times \mathbb{P}^{1}$ correspond to the two families of planes on $Q$ which pass through $p_{0}$. We shall distinguish them from each other, so that $\pi: X_{1,1} \rightarrow \mathbb{P}^{1}$ is the resolution of $Q \rightarrow Q_{0} \stackrel{\pi_{1}}{\rightarrow} \mathbb{P}^{1}$, where $\pi_{1}$ is the "first" projection. In other words, the "first" family is the $\phi$-image of $\left|L_{0,1}\right|$. On the other hand, the "second" family gives rise to $\left|L_{1,-1}\right|$, whose member is the blow-up of such a plane at $p_{0}$ and contains $\sigma$ as the (-1)-curve. The composition

$$
X_{1,1} \stackrel{\phi}{\rightarrow} Q_{\stackrel{f}{\rightarrow}}^{\rightarrow} Q_{0} \simeq \mathbb{P}^{1} \times \mathbb{P}^{1}
$$

is given by the relative projection from $\sigma$ of the $\mathbb{P}^{2}$-bundle $X_{1,1} / \mathbb{P}^{1}$.

In order to describe $\operatorname{Aut}\left(X_{1,1}\right)$, consider the blow-up $\hat{Q} \rightarrow X_{1,1}$ along $\sigma$. This is the blow-up of $Q$ at $p_{0}$ and so is the $\mathbb{P}^{1}$-bundle $\mathbb{P}\left(\mathcal{O}_{Q_{0}}(1) \oplus \mathcal{O}_{Q_{0}}\right)$ over $Q_{0}$ with exceptional divisor $\mathbb{P} \mathcal{O}_{Q_{0}}(1)$. As in (2.2), we have the exact sequence

$$
1 \rightarrow \operatorname{Aut}\left(\mathcal{O}_{Q_{0}}(1) \oplus \mathcal{O}_{Q_{0}}\right) / \mathbb{C}^{\times} \rightarrow \operatorname{Aut}(\hat{Q}) \rightarrow \operatorname{Aut}\left(Q_{0}\right) \rightarrow 1 .
$$

We may identify the quotient group $\operatorname{Aut}\left(\mathcal{O}_{Q_{0}}(1) \oplus \mathcal{O}_{Q_{0}}\right) / \mathbb{C}^{\times}$with the subgroup $R$ of $\operatorname{Aut}\left(\mathcal{O}_{Q_{0}}(1) \oplus\right.$ $\left.\mathcal{O}_{Q_{0}}\right)$ consisting of isomorphisms of the form

$$
\left(\begin{array}{ll}
\alpha & s \\
0 & 1
\end{array}\right),
$$

where $\alpha \in \mathbb{C}^{\times}$and $s \in \operatorname{Hom}\left(\mathcal{O}_{Q_{0}}, \mathcal{O}_{Q_{0}}(1)\right)$. In particular, $R \simeq \mathbb{C}^{\times} \ltimes H^{0}\left(\mathcal{O}_{Q_{0}}(1)\right)$. Now $\operatorname{Aut}\left(X_{1,1}\right)$ is the identity component of $\operatorname{Aut}(Q)=\operatorname{Aut}(\hat{Q})$. We have its natural $\mathbb{Z} / 2 \times \mathbb{Z} / 2$-covering

$$
\tilde{G}^{\prime}=\left(\mathrm{SL}_{2} \times \mathrm{SL}_{2}\right) \ltimes R \simeq\left(\mathrm{SL}_{2} \times \mathrm{SL}_{2} \times \mathbb{C}^{\times}\right) \ltimes V_{1,1} .
$$

Dividing $\tilde{G}^{\prime}$ by $(1,-1,-1) \in\left(\mathrm{SL}_{2}\right)^{2} \times \mathbb{C}^{\times}$, we obtain a double cover $\tilde{G}$ of $\operatorname{Aut}\left(X_{1,1}\right)$ isomorphic to $\left(\mathrm{SL}_{2} \times \mathrm{GL}_{2}\right) \ltimes V_{1,1}$. The kernel of the projection $\tilde{G} \rightarrow \operatorname{Aut}\left(X_{1,1}\right)$ is generated by $(-1,-1) \in$ $\mathrm{SL}_{2} \times \mathrm{GL}_{2}$.

Every line bundle on $X_{1,1}$ is obtained as the extension of one on $X_{1,1} \backslash \sigma=Q \backslash p_{0}$, which in turn is the pullback by $f$ of one on $Q_{0}$. Explicitly, we have

$$
L_{a, b} \simeq f^{*} \mathcal{O}_{Q_{0}}(b, 0) \otimes \mathcal{O}_{Q}(a) \simeq f^{*} \mathcal{O}_{Q_{0}}(a+b, a)
$$

over $Q \backslash p_{0}$.

As in $\S 2.3, H^{0}\left(L_{a, b}\right)$ is a $\tilde{G}^{\prime}$-representation and has the invariant filtration

$$
0 \subset F_{a} \subset F_{a-1} \subset \cdots \subset F_{1} \subset H^{0}\left(L_{a, b}\right),
$$

where $F_{i}$ is the space of sections vanishing to order $\geqslant i$ along $\sigma$. To be more explicit, we take bi-homogeneous coordinates $\left(\left[X_{0}, X_{1}\right],\left[Y_{0}, Y_{1}\right]\right)$ of $Q_{0}$ and homogeneous coordinates $\left[Z, Z_{00}, Z_{01}, Z_{10}, Z_{11}\right]$ of $\mathbb{P}^{4}$, where $Z_{i j}=X_{i} Y_{j}$ and $p_{0}=[1,0, \cdots, 0]$. By (2.5) we may identify $H^{0}\left(L_{a, b}\right)$ with

$$
\bigoplus_{i=0}^{a} V_{b+i}\left(X_{0}, X_{1}\right) \otimes V_{i}\left(Y_{0}, Y_{1}\right) Z^{a-i}
$$

This expression is the irreducible decomposition under the subgroup $\left(\mathrm{SL}_{2}\right)^{2} \subset \tilde{G}^{\prime}$; the $i$-th summand is isomorphic to $V_{b+i, i}$. We then have $F_{i}=\oplus_{j \geqslant i} V_{b+j, j}$. The torus $\mathbb{C}^{\times}$acts on $V_{b+i, i}$ 


\section{SHOUHEI MA}

by weight $i-a$. (Tensoring (2.6) with the weight $a$ scalar representation of $\mathbb{C}^{\times}$, we obtain a $\tilde{G}$-representation on $H^{0}\left(L_{a, b}\right)$.) The unipotent radical $V_{1,1} \ni h$ acts by the multiplication maps

$$
\cdot \exp (h): V_{b+i, i} \rightarrow \underset{d=0}{a-i} V_{b+i+d, i+d}
$$

where $\exp (h)=\left(1, h, h^{\otimes 2} / 2, \cdots\right) \in \oplus_{d \geqslant 0} V_{d, d}$. In particular, the quotient representation $H^{0}\left(L_{a, b}\right) / F_{i}$ is isomorphic to $H^{0}\left(L_{i-1, b}\right)$. Geometrically the quotient map $H^{0}\left(L_{a, b}\right) \rightarrow H^{0}\left(L_{a, b}\right) / F_{i}$ gives the Taylor expansions up to $(i-1)$ th order of the sections of $L_{a, b}$ along the exceptional divisor $E$ of $\hat{Q}$. Here notice that $\left.L_{a, b}\right|_{E} \otimes\left(N_{E / \hat{Q}}\right)^{-k} \simeq \mathcal{O}_{E}(b+k, k)$.

Lemma 2.5. The line bundle $L_{a, b}$ is $\operatorname{Aut}\left(X_{1,1}\right)$-linearized when $b$ is even.

Proof. We have natural Aut $\left(X_{1,1}\right)$-linearizations on $L_{0,-2}=\pi^{*} K_{\mathbb{P}^{1}}, L_{-2,0}=f^{*} K_{Q_{0}}$ and $L_{-3,0}=K_{X_{1,1}}$. When $b$ is even, $L_{a, b}$ can be written as a tensor product of these bundles.

Remark 2.6. More generally, on $X_{e, f}$ with $e \neq 0($ resp. $0=e<f)$, the bundle $L_{a, b}$ is $\operatorname{Aut}\left(X_{e, f}\right)$ linearized if $b$ (resp. $a f+b$ ) is even.

\section{Tetragonal loci}

In this section we follow Schreyer's description [Sch86] of tetragonal curves to derive a birational model of the tetragonal locus $\mathcal{T}_{g}$ as a quotient space. Notice that we are assuming that $g \geqslant 7$. First recall some basic facts:

- a tetragonal curve $C$ is not trigonal;

- when $C$ is general in $\mathcal{T}_{g}$, its tetragonal pencil $C \rightarrow \mathbb{P}^{1}$ is unique;

- if $g \geqslant 10, C$ has a unique tetragonal pencil precisely when $C$ is not bielliptic;

- $\mathcal{T}_{g}$ is irreducible of dimension $2 g+3$.

The first three properties can be seen by looking at the product $C \rightarrow \mathbb{P}^{1} \times \mathbb{P}^{1}$ of two pencils. See [AC81] and the references therein for the last property.

Now let $\pi: C \rightarrow \mathbb{P}^{1}$ be a tetragonal map. We regard $C$ as canonically embedded in $\mathbb{P}^{g-1}$. For each $p \in \mathbb{P}^{1}$, the (possibly infinitely near) four points $\pi^{-1}(p)$ span a plane in $\mathbb{P}^{g-1}$ by RiemannRoch. The threefold swept out by those planes is a rational normal scroll; we may write it as $\phi_{L_{1, n}}\left(X_{e, f}\right)$ for some $0 \leqslant e \leqslant f$ and $n \geqslant 0$. If we view $C$ as a curve on $X_{e, f}$, it turns out to be a complete intersection of two surfaces in $\left|L_{2, b}\right|,\left|L_{2, c}\right|$ for some $b \leqslant c$ ([Sch86]). Comparing the adjunction formula for $C$ with the relation $\left.L_{1, n}\right|_{C} \simeq K_{C}$, we see that

$$
n=b+c+e+f-2 .
$$

Calculating $\operatorname{deg} K_{C}=\left(L_{1, n} . L_{2, b} \cdot L_{2, c}\right)$, we obtain

$$
g=4(e+f)+3(b+c-1),
$$

which imposes a relation between $(e, f)$ and $(b, c)$.

Let $\hat{\mathcal{T}}_{g}$ be the moduli space of tetragonal curves of genus $g \geqslant 7$ given with a tetragonal pencil $C \rightarrow \mathbb{P}^{1}$. The natural projection $\hat{\mathcal{T}}_{g} \rightarrow \mathcal{T}_{g}$ is birational. For $0 \leqslant e \leqslant f$ and $b \leqslant c$, let $\hat{\mathcal{T}}_{g}(e, f ; b, c) \subset \hat{\mathcal{T}}_{g}$ be the locus of those $C \rightarrow \mathbb{P}^{1}$ which lie on $X_{e, f}$ as a complete intersection of surfaces in $\left|L_{2, b}\right|$ and $\left|L_{2, c}\right|$. Then we have the stratification

$$
\hat{\mathcal{T}}_{g}=\underset{(e, f ; b, c)}{\sqcup} \hat{\mathcal{T}}_{g}(e, f ; b, c),
$$




\section{RATIONALITY OF SOME TETRAGONAL LOCI}

where $(e, f)$ and $(b, c)$ satisfy (3.1). This presentation still includes many empty strata (see [Sch86]), but we do not mind this redundancy here.

Since the embedding $C \subset X_{e, f}$ is canonical, we find that each stratum is the quotient by $\operatorname{Aut}\left(X_{e, f}\right)$ of the parameter space of those complete intersection curves. More precisely, when $b=c$, we have

$$
\hat{\mathcal{T}}_{g}(e, f ; b, b) \sim \mathbb{G}\left(1,\left|L_{2, b}\right|\right) / \operatorname{Aut}\left(X_{e, f}\right),
$$

where $\mathbb{G}\left(1,\left|L_{2, b}\right|\right)$ is the Grassmannian of pencils in $\left|L_{2, b}\right|$. On the other hand, when $b<c$, the surface $S \in\left|L_{2, b}\right|$ containing $C$ is unique, while those in $\left|L_{2, c}\right|$ are unique up to $S+\left|L_{0, c-b}\right|$. To express this situation, let $\mathcal{L} \rightarrow\left|L_{2, b}\right|$ be the tautological bundle and let $\mathcal{E} \rightarrow\left|L_{2, b}\right|$ be the quotient bundle

$$
\mathcal{E}=\underline{H^{0}\left(L_{2, c}\right)} / \mathcal{L} \otimes H^{0}\left(L_{0, c-b}\right),
$$

where $H^{0}\left(L_{2, c}\right)$ is the product bundle $H^{0}\left(L_{2, c}\right) \times\left|L_{2, b}\right|$, and the bundle homomorphism $\mathcal{L} \otimes$ $H^{0}\left(L_{0, c-b}\right) \rightarrow H^{0}\left(L_{2, c}\right)$ is induced by the multiplication map $H^{0}\left(L_{2, b}\right) \times H^{0}\left(L_{0, c-b}\right) \rightarrow H^{0}\left(L_{2, c}\right)$. Then we have

$$
\hat{\mathcal{T}}_{g}(e, f ; b, c) \sim \mathbb{P} \mathcal{E} / \operatorname{Aut}\left(X_{e, f}\right) .
$$

In order to study the birational type of $\mathcal{T}_{g}$, we want to identify the largest stratum in (3.2). This was done by Del Centina and Gimigliano in [dCG93]. The result depends on the congruence of $g$ modulo 6 and is summarized as follows (see also [CdC04, §3]).

Proposition 3.1. Let $\mathcal{E}$ be the bundle defined in (3.3) with $c=b+1$.

(0) When $g \equiv 0(6)$, write $g=6 b$. Then $\left(L_{2, b}, L_{2, b+1}\right)$ complete intersections in $X_{0,0}=\mathbb{P}^{1} \times \mathbb{P}^{2}$ give the largest stratum. Hence $\mathcal{T}_{6 b} \sim \mathbb{P} \mathcal{E} / \mathrm{PGL}_{2} \times \mathrm{PGL}_{3}$.

(1) When $g \equiv 1(6)$, write $g=6 b+1$. Then $\left(L_{2, b}, L_{2, b}\right)$ complete intersections in $X_{0,1}$ give the largest stratum. Thus $\mathcal{T}_{6 b+1} \sim \mathbb{G}\left(1,\left|L_{2, b}\right|\right) / \operatorname{Aut}\left(X_{0,1}\right)$.

(2) When $g \equiv 2(6)$, write $g=6 b+8$. Then $\left(L_{2, b}, L_{2, b+1}\right)$ complete intersections in $X_{1,1}$ give the largest stratum. Hence $\mathcal{T}_{6 b+8} \sim \mathbb{P E} / \operatorname{Aut}\left(X_{1,1}\right)$.

(3) When $g \equiv 3(6)$, write $g=6 b-3$. Then $\left(L_{2, b}, L_{2, b}\right)$ complete intersections in $X_{0,0}$ give the largest stratum. Thus $\mathcal{T}_{6 b-3} \sim \mathbb{G}\left(1,\left|L_{2, b}\right|\right) / \mathrm{PGL}_{2} \times \mathrm{PGL}_{3}$.

(4) When $g \equiv 4(6)$, write $g=6 b+4$. Then $\left(L_{2, b}, L_{2, b+1}\right)$ complete intersections in $X_{0,1}$ give the largest stratum. Hence $\mathcal{T}_{6 b+4} \sim \mathbb{P E} / \operatorname{Aut}\left(X_{0,1}\right)$.

(5) When $g \equiv 5(6)$, write $g=6 b+5$. Then $\left(L_{2, b}, L_{2, b}\right)$ complete intersections in $X_{1,1}$ give the largest stratum. Thus $\mathcal{T}_{6 b+5} \sim \mathbb{G}\left(1,\left|L_{2, b}\right|\right) / \operatorname{Aut}\left(X_{1,1}\right)$.

Proof. Let us give a self-contained argument. It is sufficient to check that the above quotients have dimension $2 g+3$, and this follows from the formulae

$$
\begin{gathered}
\operatorname{dim} \operatorname{Aut}\left(X_{0,0}\right)=\operatorname{dim} \operatorname{Aut}\left(X_{0,1}\right)=\operatorname{dim} \operatorname{Aut}\left(X_{1,1}\right)=11, \\
h^{0}\left(L_{2, b}\right)=4(e+f)+6(b+1),
\end{gathered}
$$

from $\S 2$.

In $\S 5-\S 7$, we will use these descriptions of $\mathcal{T}_{g}$ to prove Theorem 1.1. In order to have $\operatorname{Aut}\left(X_{e, f}\right)$-linearizations on some vector bundles, we were forced to assume $b$ to be even in cases (1), (3), (5), and odd in cases (0), (2), (4). This caused the further mod 12 classification in Theorem 1.1. 


\section{SHOUHEI MA}

\section{Supplementary techniques for rationality}

In this section we collect some techniques for proving the rationality of quotient varieties that supplement the basic ones as in [Böh09] and that will be used repeatedly in the rest of this article. We encourage the reader to skip this section for the moment and return to it when necessary. Most of this section is more or less standard, but for the convenience of the reader we have sketched some proofs.

\subsection{Quotients of Grassmannians}

Let $G$ be an algebraic group and let $V$ be a $G$-representation. We denote by $G_{0} \subset G$ the subgroup of elements which act on $V$ by scalar multiplication, and set $\bar{G}=G / G_{0}$. Let $G(a, V)=\mathbb{G}(a-$ $1, \mathbb{P} V)$ be the Grassmannian of $a$-dimensional linear subspaces in $V$. In view of Proposition 3.1, we will be interested in the problem whether the quotient $G(a, V) / G$ is rational, or at least stably rational of small level. First notice that we have a natural birational identification

$$
G(a, V) / G \sim \operatorname{Hom}\left(\mathbb{C}^{a}, V\right) / \mathrm{GL}_{a} \times G=\left(\left(\mathbb{C}^{a}\right)^{\vee} \otimes V\right) / \mathrm{GL}_{a} \times G,
$$

so that the problem could be reduced to the case of linear quotients.

To prove the stable rationality of $G(a, V) / G$, it is useful to consider the universal subbundle $\mathcal{E} \rightarrow G(a, V)$ of rank $a$, which is $G$-linearized. Its projectivization is viewed as the correspondence

$$
\mathbb{P} \mathcal{E}=\{(P,[v]) \in G(a, V) \times \mathbb{P} V \mid \mathbb{C} v \subset P\}
$$

between $G(a, V)$ and $\mathbb{P} V$. The second projection $\mathbb{P} \mathcal{E} \rightarrow \mathbb{P} V$ is identified with the relative Grassmannian $G(a-1, \mathcal{F})$ over $\mathbb{P} V$, where $\mathcal{F} \rightarrow \mathbb{P} V$ is the universal quotient bundle of rank $\operatorname{dim} V-1$.

Lemma 4.1. Suppose that $\bar{G}$ acts on $G(a, V)$ almost freely and that $G_{0}$ acts on $\mathcal{E} \otimes(\operatorname{det} \mathcal{E})^{d}$ trivially for some $d \in \mathbb{Z}$.

(1) If furthermore $\bar{G}$ acts on $\mathbb{P} V$ almost freely, then

$$
\mathbb{P}^{a-1} \times(G(a, V) / G) \sim G(a-1, \operatorname{dim} V-1) \times(\mathbb{P} V / G) .
$$

(2) If $G$ acts on $\mathbb{P} V$ almost transitively with $H \subset G$ the stabilizer of a general point $[v] \in \mathbb{P} V$, then

$$
\mathbb{P}^{a-1} \times(G(a, V) / G) \sim G(a-1, V / \mathbb{C} v) / H .
$$

Proof. Note that we have a canonical identification $\mathbb{P} \mathcal{E}=\mathbb{P}\left(\mathcal{E} \otimes(\operatorname{det} \mathcal{E})^{d}\right)$. Using the no-name lemma for the bundle $\mathcal{E} \otimes(\operatorname{det} \mathcal{E})^{d}$ which is $\bar{G}$-linearized, we obtain $\mathbb{P} \mathcal{E} / G \sim \mathbb{P}^{a-1} \times(G(a, V) / G)$. On the other hand, the bundle $\mathcal{F} \otimes \mathcal{O}_{\mathbb{P} V}(1)$ over $\mathbb{P} V$ is always $\bar{G}$-linearized, and we have $G(a-1, \mathcal{F})=G\left(a-1, \mathcal{F} \otimes \mathcal{O}_{\mathbb{P} V}(1)\right)$. Now statement $(1)$ is a consequence of the no-name lemma applied to $\mathcal{F} \otimes \mathcal{O}_{\mathbb{P} V}(1)$, while statement (2) follows from the slice method for the projection $G(a-1, \mathcal{F}) \rightarrow \mathbb{P} V$.

Next consider the situation where we have a surjective $G$-homomorphism $f: V \rightarrow W$ to another $G$-representation $W$. Notice that we are not assuming $V$ to be completely reducible. We have a natural dominant map

$$
G(a, V) \rightarrow G(a, W)
$$

whose fiber over $P \in G(a, W)$ is an open subset of $G\left(a, f^{-1}(P)\right)$. Let $\mathcal{G} \rightarrow G(a, W)$ be the universal subbundle for $G(a, W)$, and let $\mathcal{H} \rightarrow G(a, W)$ be the vector bundle obtained as the inverse image of $\mathcal{G}$ by the bundle homomorphism $\underline{f}: \underline{V} \rightarrow \underline{W}$ over $G(a, W)$. Then (4.2) induces 


\section{RATIONALITY OF SOME TETRAGONAL LOCI}

a $G$-equivariant birational map

$$
G(a, V) \rightarrow G(a, \mathcal{H})
$$

to the relative Grassmannian $G(a, \mathcal{H})$. As in the proof of Lemma 4.1, we obtain the following result using the no-name lemma.

Lemma 4.2. Suppose that $\bar{G}$ acts on $G(a, W)$ almost freely and that $G_{0}$ acts on $\mathcal{H} \otimes(\operatorname{det} \mathcal{G})^{d}$ trivially for some $d \in \mathbb{Z}$. Then, setting $n_{0}=\operatorname{dim} V-\operatorname{dim} W$, we have

$$
G(a, V) / G \sim G\left(a, n_{0}+a\right) \times(G(a, W) / G) .
$$

This can be seen as a Grassmannian version of the no-name method.

In the above lemmas, we are required to check the almost-freeness of an action on a Grassmannian. In many cases it follows from the following observation. (This can also essentially be found in the proof of Proposition 1.3.2.10 in [Böh09].)

Lemma 4.3. Let an algebraic group $G$ act on a projective space $\mathbb{P}^{n}$ almost freely. If $a<n-\operatorname{dim} G$, then $G$ acts on $\mathbb{G}\left(a, \mathbb{P}^{n}\right)$ almost freely.

Proof. Let $p \in \mathbb{P}^{n}$ be a general point. It suffices to show that a general $a$-plane $P \subset \mathbb{P}^{n}$ through $p$ is not stabilized by any element of $G$. Consider the projection $\pi: \mathbb{P}^{n} \rightarrow \mathbb{P}^{n-1}$ from $p$. Since $\pi(G \cdot p \backslash p) \subset \mathbb{P}^{n-1}$ has dimension at most $\operatorname{dim} G$, a general $(a-1)$-plane $P^{\prime} \subset \mathbb{P}^{n-1}$ is disjoint from $\pi(G \cdot p \backslash p)$ by our assumption that $a<n-\operatorname{dim} G$. This means that $P \cap(G \cdot p)=\{p\}$. Now if $g \in G$ stabilizes $P$, then we have $g(p)=g(P \cap(G \cdot p))=P \cap(G \cdot p)=p$, so that $g=$ id.

\subsection{Representations of product groups}

We can use quotients of Grassmannians for the rationality problem for representations of product groups (see[Ma13b] for more detail). Let $G, H$ be algebraic groups and let $V, W$ be representations of $G, H$, respectively. Then $V \otimes W$ is a representation of $G \times H$. We assume that $\operatorname{dim} V<\operatorname{dim} W$. Identifying $V \otimes W$ with $\operatorname{Hom}\left(V^{\vee}, W\right)$, we consider the map

$$
\operatorname{Hom}\left(V^{\vee}, W\right) \rightarrow G(\operatorname{dim} V, W)
$$

that sends a homomorphism to its image. Let $\mathcal{E} \rightarrow G(\operatorname{dim} V, W)$ be the universal subbundle. Then (4.3) induces a birational map

$$
V \otimes W \rightarrow V \otimes \mathcal{E}
$$

to the vector bundle $V \otimes \mathcal{E}=\operatorname{Hom}\left(V^{\vee}, \mathcal{E}\right)$ over $G(\operatorname{dim} V, W)$. Here $H$ acts on $\mathcal{E}$ equivariantly and $G$ acts on $V$ fiberwisely. As in $\S 4.1$, let $H_{0}=\operatorname{Ker}(H \rightarrow \operatorname{PGL}(W))$ and let $\bar{H}=H / H_{0}$. By the no-name method we then obtain the following result.

Lemma 4.4 [Ma13b]. Suppose that $\bar{H}$ acts on $G(\operatorname{dim} V, W)$ almost freely and that $H_{0}$ acts on $\mathcal{E} \otimes(\operatorname{det} \mathcal{E})^{d}$ trivially for some $d \in \mathbb{Z}$. Then

$$
\mathbb{P}(V \otimes W) / G \times H \sim\left(\mathbb{P}\left(V^{\oplus \operatorname{dim} V}\right) / G\right) \times(G(\operatorname{dim} V, W) / H) .
$$

\section{The case of $\mathbb{P}^{1} \times \mathbb{P}^{2}$}

We begin the proof of Theorem 1.1 with the cases $g \equiv 6,9(12)$, where the basic $\mathbb{P}^{2}$-bundle is $\mathbb{P}^{1} \times \mathbb{P}^{2}$. We shall use the standard notation $\mathcal{O}_{\mathbb{P}^{1} \times \mathbb{P}^{2}}(b, a)$ for line bundles on $\mathbb{P}^{1} \times \mathbb{P}^{2}$, rather than the notation $L_{a, b}$ used in $\S 2$. The automorphism group of $\mathbb{P}^{1} \times \mathbb{P}^{2}$ is $\mathrm{PGL}_{2} \times \mathrm{PGL}_{3}$. It is useful 


\section{SHOUHEI MA}

to also consider $\mathrm{SL}_{2} \times \mathrm{SL}_{3}$, because any line bundle $\mathcal{O}_{\mathbb{P}^{1} \times \mathbb{P}^{2}}(b, a)$ is $\mathrm{SL}_{2} \times \mathrm{SL}_{3}$-linearized. The natural projection $\mathrm{SL}_{2} \times \mathrm{SL}_{3} \rightarrow \mathrm{PGL}_{2} \times \mathrm{PGL}_{3}$ has kernel $\mathbb{Z} / 6$ generated by

$$
\zeta=\left(-1, e^{2 \pi i / 3}\right) \in \mathrm{SL}_{2} \times \mathrm{SL}_{3} .
$$

This element acts on $\mathcal{O}_{\mathbb{P}^{1} \times \mathbb{P}^{2}}(b, a)$ by multiplication by $\left(e^{\pi i / 3}\right)^{3 b-2 a}$.

In the remainder of this section we use the abbreviation $W_{a}$ for the $\mathrm{SL}_{3}$-representation $H^{0}\left(\mathcal{O}_{\mathbb{P}^{2}}(a)\right)$. Thus we have $H^{0}\left(\mathcal{O}_{\mathbb{P}^{1} \times \mathbb{P}^{2}}(b, a)\right) \simeq V_{b} \otimes W_{a}$ as an $\mathrm{SL}_{2} \times \mathrm{SL}_{3}$-representation.

\subsection{The case $g \equiv 6(12)$}

Let $b \geqslant 3$ be an odd number. Let $\mathcal{L} \rightarrow \mathbb{P}\left(V_{b} \otimes W_{2}\right)$ be the tautological bundle, and let $\mathcal{E} \rightarrow$ $\mathbb{P}\left(V_{b} \otimes W_{2}\right)$ be the bundle $V_{b+1} \otimes W_{2} / \mathcal{L} \otimes V_{1}$ as defined in Proposition 3.1. The bundles $\mathcal{L}$ and $\mathcal{E}$ are $\mathrm{SL}_{2} \times \mathrm{SL}_{3}$-linearized, where $\zeta$ acts by $\left(e^{\pi i / 3}\right)^{3 b-4}$ and $\left(e^{\pi i / 3}\right)^{3 b-1}$, respectively. Recall that by Proposition 3.1, $\mathbb{P} \mathcal{E} / \mathrm{SL}_{2} \times \mathrm{SL}_{3}$ is birational to the tetragonal locus of genus $6 b \equiv 6$ (12).

Lemma 5.1. The group $\mathrm{PGL}_{2} \times \mathrm{PGL}_{3}$ acts on $\mathbb{P}\left(V_{b} \otimes W_{2}\right)$ almost freely.

Proof. It is known that a general member $S$ of $\left|\mathcal{O}_{\mathbb{P}^{1} \times \mathbb{P}^{2}}(b, 2)\right|$ is the blow-up of $\mathbb{P}^{2}$ at $3 b+1$ points in general position (for example, put $s=3 b$ and $n=s-1$ in [dCG93, §2]). Since $3 b+1 \geqslant 10, S$ has no nontrivial automorphism (see [Koi88]). If $g \in \mathrm{PGL}_{2} \times \mathrm{PGL}_{3}$ acts trivially on $S$, then it also does so on $\mathbb{P}^{1} \times \mathbb{P}^{2}$.

Proposition 5.2. The quotient $\mathbb{P} \mathcal{E} / \mathrm{SL}_{2} \times \mathrm{SL}_{3}$ is rational.

Proof. Since $\zeta$ acts on $\mathcal{E} \otimes \mathcal{L}^{2}$ by multiplication by $\left(e^{\pi i / 3}\right)^{9 b-9}=(-1)^{b-1}=1$, the bundle $\mathcal{E} \otimes \mathcal{L}^{2}$ is $\mathrm{PGL}_{2} \times \mathrm{PGL}_{3}$-linearized. We identify $\mathbb{P} \mathcal{E}$ with $\mathbb{P}\left(\mathcal{E} \otimes \mathcal{L}^{2}\right)$ and apply the no-name lemma to the latter, which is possible by the above lemma. Then we have

$$
\mathbb{P} \mathcal{E} / \mathrm{PGL}_{2} \times \mathrm{PGL}_{3} \sim \mathbb{P}^{6 b+9} \times\left(\mathbb{P}\left(V_{b} \otimes W_{2}\right) / \mathrm{PGL}_{2} \times \mathrm{PGL}_{3}\right) .
$$

In order to show that $\mathbb{P}\left(V_{b} \otimes W_{2}\right) / \mathrm{SL}_{2} \times \mathrm{SL}_{3}$ is stably rational of level $6 b+9$, we consider the product $U=\mathbb{P}\left(V_{b} \otimes W_{2}\right) \times \mathbb{P}\left(V_{5} \otimes W_{1}\right)$. Let $\mathcal{L}^{\prime} \rightarrow \mathbb{P}\left(V_{5} \otimes W_{1}\right)$ be the tautological bundle. The first projection $U \rightarrow \mathbb{P}\left(V_{b} \otimes W_{2}\right)$ may be identified with the projective bundle $\mathbb{P}\left(V_{5} \otimes W_{1} \otimes \mathcal{L}\right)$, while the second $U \rightarrow \mathbb{P}\left(V_{5} \otimes W_{1}\right)$ may be identified with $\mathbb{P}\left(V_{b} \otimes W_{2} \otimes \mathcal{L}^{\prime}\right)$. Since $\zeta$ acts trivially on both $V_{5} \otimes W_{1} \otimes \mathcal{L}$ and $V_{b} \otimes W_{2} \otimes \mathcal{L}^{\prime}$, these bundles are $\mathrm{PGL}_{2} \times \mathrm{PGL}_{3}$-linearized. Applying the no-name lemma to the two projections, we obtain

$$
\begin{aligned}
U / \mathrm{PGL}_{2} \times \mathrm{PGL}_{3} & \sim \mathbb{P}^{17} \times\left(\mathbb{P}\left(V_{b} \otimes W_{2}\right) / \mathrm{PGL}_{2} \times \mathrm{PGL}_{3}\right) \\
& \sim \mathbb{P}^{6 b+5} \times\left(\mathbb{P}\left(V_{5} \otimes W_{1}\right) / \mathrm{PGL}_{2} \times \mathrm{PGL}_{3}\right) .
\end{aligned}
$$

Here $\mathrm{PGL}_{2} \times \mathrm{PGL}_{3}$ acts on $\mathbb{P}\left(V_{5} \otimes W_{1}\right)$ almost freely because we have (4.1) and $\mathrm{PGL}_{2}$ acts on $G\left(3, V_{5}\right)$ almost freely ([Ma13b, Lemma 2.7]). Thus the problem is reduced to showing the stable rationality of level $6 b+5$ of

$$
\mathbb{P}\left(V_{5} \otimes W_{1}\right) / \mathrm{SL}_{2} \times \mathrm{SL}_{3} \sim G\left(3, V_{5}\right) / \mathrm{SL}_{2} .
$$

This in turn follows from Lemma 4.1 (1) and the rationality of $\mathbb{P} V_{5} / \mathrm{SL}_{2}$ (which has dimension 2).

\subsection{The $\mathrm{PGL}_{2} \times \mathrm{PGL}_{3}$-action on $\mathbb{P}\left(V_{1} \otimes W_{2}\right)$}

Before going to the case $g \equiv 9$ (12), we study here the action of $\mathrm{PGL}_{2} \times \mathrm{PGL}_{3}$ on $\mathbb{P}\left(V_{1} \otimes W_{2}\right)$. Let $\mathcal{E} \rightarrow \mathbb{G}\left(1, \mathbb{P} W_{2}\right)$ be the universal subbundle and consider the birational equivalence $\mathbb{P}\left(V_{1} \otimes W_{2}\right) \sim$ 


\section{RATIONALITY OF SOME TETRAGONAL LOCI}

$\mathbb{P}\left(V_{1} \otimes \mathcal{E}\right)$ in (4.4). Then $\mathrm{PGL}_{2}$ acts on each fiber of $\mathbb{P}\left(V_{1} \otimes \mathcal{E}\right) \rightarrow \mathbb{G}\left(1, \mathbb{P} W_{2}\right)$ almost freely and almost transitively. On the other hand, since a general conic pencil on $\mathbb{P}^{2}$ is determined by its four base points in general position, we see that $\mathrm{PGL}_{3}$ acts on $\mathbb{G}\left(1, \mathbb{P} W_{2}\right)$ almost transitively and the stabilizer of a general pencil is the permutation group of its four base points. Thus the $\mathrm{PGL}_{2} \times \mathrm{PGL}_{3}$-action on $\mathbb{P}\left(V_{1} \otimes W_{2}\right)$ is almost transitive, with $\widetilde{\Xi}_{4}$ the stabilizer of a general point. Let us study how this $\mathfrak{\Xi}_{4}$ acts on $\mathbb{P}\left(V_{1} \otimes W_{2}\right)$.

Recall first that the irreducible representations of $\mathfrak{\subseteq}_{4}$ are the following five ([Ser77]):

- the trivial representation $\chi_{0}$

- the sign representation $\epsilon$

- the three-dimensional standard representation $\psi$

- the tensor product $\epsilon \psi=\epsilon \otimes \psi$

- the two-dimensional standard representation $\theta$ of $\mathfrak{\Xi}_{3}$, where we regard $\mathfrak{\Xi}_{3}$ as the quotient of $\mathfrak{\Xi}_{4}$ by the Klein four-group.

Now we may normalize the four base points on $\mathbb{P}^{2}$ so that $\mathbb{P}^{2}=\mathbb{P}(\psi)$ as an $\mathfrak{\Xi}_{4}$-space. Then $\mathbb{P}\left(W_{2}\right)=\mathbb{P}\left(\operatorname{Sym}^{2} \psi^{\vee}\right)$, and we have the decomposition

$$
\operatorname{Sym}^{2} \psi^{\vee} \simeq \operatorname{Sym}^{2} \psi \simeq \chi_{0} \oplus \theta \oplus \psi \text {. }
$$

The conic pencil associated with the four points is $\mathbb{P}(\theta) \subset \mathbb{P} W_{2}$. Since the fiber of $\mathcal{E} \rightarrow \mathbb{G}\left(1, \mathbb{P} W_{2}\right)$ over the point $\mathbb{P}(\theta) \in \mathbb{G}\left(1, \mathbb{P} W_{2}\right)$ is $\theta$ itself, we see that $\mathbb{P} V_{1} \simeq \mathbb{P}\left(\theta^{\vee}\right)$ as an $\mathfrak{S}_{4}$-space. Hence $\mathbb{P}\left(V_{1} \otimes W_{2}\right) \simeq \mathbb{P}\left(\theta^{\vee} \otimes\left(\chi_{0} \oplus \theta \oplus \psi\right)\right)$. Noticing that $\theta^{\vee} \simeq \theta, \theta^{\vee} \otimes \theta \simeq \chi_{0} \oplus \epsilon \oplus \theta$ and $\theta^{\vee} \otimes \psi \simeq \psi \oplus \epsilon \psi$, we summarize the argument as follows.

Lemma 5.3. The group $\mathrm{PGL}_{2} \times \mathrm{PGL}_{3}$ acts on $\mathbb{P}\left(V_{1} \otimes W_{2}\right)$ almost transitively, with stabilizer of a general point $[v]$ isomorphic to $\mathfrak{\Xi}_{4}$. As an $\mathfrak{\Xi}_{4}$-space,

$$
\mathbb{P}\left(V_{1} \otimes W_{2}\right) \simeq \mathbb{P}\left(\chi_{0} \oplus \epsilon \oplus \theta^{\oplus 2} \oplus \psi \oplus \epsilon \psi\right),
$$

where $[v]$ is $\mathbb{P}\left(\chi_{0}\right) \in \mathbb{P}\left(V_{1} \otimes W_{2}\right)$.

For later use, we remark that the following fact is well known: it is a simple application of the no-name method.

Proposition 5.4. For any $\mathfrak{\subseteq}_{4}$-representation $V$ the quotient $\mathbb{P} V / \mathfrak{\subseteq}_{4}$ is rational.

5.3 The case $g \equiv 9(12)$

Let $b>0$ be an even number. By Proposition 3.1, the quotient $G\left(2, V_{b} \otimes W_{2}\right) / \mathrm{SL}_{2} \times \mathrm{SL}_{3}$ is birational to the tetragonal locus of genus $6 b-3 \equiv 9(12)$. We shall prove the following result.

Proposition 5.5. The quotient $G\left(2, V_{b} \otimes W_{2}\right) / \mathrm{SL}_{2} \times \mathrm{SL}_{3}$ is rational for $b \neq 2,8$.

To begin, we use (4.1) to rewrite $G\left(2, V_{b} \otimes W_{2}\right) / \mathrm{SL}_{2} \times \mathrm{SL}_{3}$ as

$$
\mathbb{P}\left(V_{1} \otimes V_{b} \otimes W_{2}\right) / \mathrm{SL}_{2} \times \mathrm{SL}_{2} \times \mathrm{SL}_{3} .
$$

5.3.1 The case $b \geqslant 12$ When $b \geqslant 12$, we have $\operatorname{dim} V_{b}>\operatorname{dim}\left(V_{1} \otimes W_{2}\right)=12$. We then want to use the method of $\S 4.2$ for $V_{1} \otimes V_{b} \otimes W_{2}=\left(V_{1} \otimes W_{2}\right) \otimes V_{b}$, viewed as a representation of $\left(\mathrm{SL}_{2} \times \mathrm{SL}_{3}\right) \times \mathrm{SL}_{2}$. Since $b$ is even, $V_{b}$ is a representation of $\mathrm{PGL}_{2}$, so that the universal subbundle over $G\left(12, V_{b}\right)$ is $\mathrm{PGL}_{2}$-linearized. By Lemma $4.3, \mathrm{PGL}_{2}$ acts almost freely on $G\left(12, V_{b}\right) \simeq G(b-$ 


\section{Shouhei MA}

$\left.11, V_{b}\right)$. Thus we can apply Lemma 4.4 to see that $(5.2)$ is birational to

$$
\left(\mathbb{P}\left(V_{1} \otimes W_{2}\right)^{\oplus 12} / \mathrm{SL}_{2} \times \mathrm{SL}_{3}\right) \times\left(G\left(12, V_{b}\right) / \mathrm{SL}_{2}\right) .
$$

Since $\mathbb{P} V_{b} / \mathrm{SL}_{2}$ is rational by Katsylo [Kat84a], $G\left(12, V_{b}\right) / \mathrm{SL}_{2}$ is stably rational of level 11 by Lemma 4.1 (1). Hence it remains to prove that $\mathbb{P}\left(V_{1} \otimes W_{2}\right)^{\oplus 12} / \mathrm{SL}_{2} \times \mathrm{SL}_{3}$ is rational.

By Lemma 5.3, we can apply the slice method to the projection $\mathbb{P}\left(V_{1} \otimes W_{2}\right)^{\oplus 12} \rightarrow \mathbb{P}\left(V_{1} \otimes W_{2}\right)$ to the first summand. Then we have

$$
\mathbb{P}\left(V_{1} \otimes W_{2}\right)^{\oplus 12} / \mathrm{PGL}_{2} \times \mathrm{PGL}_{3} \sim\left(V_{1} \otimes W_{2}\right)^{\oplus 11} / \Im_{4} .
$$

The right-hand side is rational by Proposition 5.4. Thus Proposition 5.5 is proved for $b \geqslant 12$.

5.3.2 The case $b=4,6$ Let $b$ be either 4 or 6 . Then $\operatorname{dim} V_{b}<\operatorname{dim}\left(V_{1} \otimes W_{2}\right)$. We shall consider $V_{1} \otimes V_{b} \otimes W_{2}$ as the $\mathrm{SL}_{2} \times\left(\mathrm{SL}_{2} \times \mathrm{SL}_{3}\right)$-representation $V_{b} \otimes\left(V_{1} \otimes W_{2}\right)$, and apply the method of $\S 4$.2. Let $\mathcal{E} \rightarrow G\left(b+1, V_{1} \otimes W_{2}\right)$ be the universal subbundle. Then $\zeta$ acts on $\mathcal{E} \otimes \operatorname{det} \mathcal{E}$ trivially in case $b=4$, and on $\mathcal{E} \otimes(\operatorname{det} \mathcal{E})^{-1}$ trivially in case $b=6$. One checks (for example, by looking at various special loci in $\left.\mathbb{P}\left(V_{1} \otimes W_{2}\right)\right)$ that $\mathrm{PGL}_{2} \times \mathrm{PGL}_{3}$ acts on $G\left(b+1, V_{1} \otimes W_{2}\right)$ almost freely. So by Lemma 4.4, the quotient (5.2) is birational to

$$
\left(\mathbb{P} V_{b}^{\oplus b+1} / \mathrm{SL}_{2}\right) \times\left(G\left(b+1, V_{1} \otimes W_{2}\right) / \mathrm{SL}_{2} \times \mathrm{SL}_{3}\right) .
$$

The first quotient $\mathbb{P} V_{b}^{\oplus b+1} / \mathrm{SL}_{2}$ is rational by Katsylo [Kat84b], so it suffices to show that $G\left(b+1, V_{1} \otimes W_{2}\right) / \mathrm{SL}_{2} \times \mathrm{SL}_{3}$ is stably rational of level $(b+1)^{2}-4$.

We regard $V_{1} \otimes W_{2}$ as an $\mathfrak{\Xi}_{4}$-representation as in the right side of (5.1) and write $V=$ $V_{1} \otimes W_{2} / \chi_{0}$. Combining Lemma 4.1 (2) and Lemma 5.3, we see that

$$
\mathbb{P}^{b} \times\left(G\left(b+1, V_{1} \otimes W_{2}\right) / \mathrm{SL}_{2} \times \mathrm{SL}_{3}\right) \sim G(b, V) / \Im_{4} .
$$

By looking at the decomposition (5.1), we can find an $\varsigma_{4}$-invariant subspace $V^{\prime} \subset V$ of dimension $b$ in either case. If $V^{\prime \prime} \subset V$ is the complementary sub- $\Im_{4}$-representation, we have the $\mathfrak{\Xi}_{4}$-invariant open set $\operatorname{Hom}\left(V^{\prime}, V^{\prime \prime}\right) \subset G(b, V)$, where $\Im_{4}$ acts on $\operatorname{Hom}\left(V^{\prime}, V^{\prime \prime}\right)$ linearly. Then $\operatorname{Hom}\left(V^{\prime}, V^{\prime \prime}\right) / \Im_{4}$ is rational by Proposition 5.4, so Proposition 5.5 is proved for $b=4,6$.

Remark 5.6. It seems that the same approach does not work for $b=2,8$, because $\zeta$ acts on $\mathcal{E} \otimes(\operatorname{det} \mathcal{E})^{d}$ nontrivially for any $d \in \mathbb{Z}$.

5.3.3 The case $b=10$ As in $\S 5.3 .2$, we consider $V_{1} \otimes V_{10} \otimes W_{2}$ as the $\mathrm{SL}_{2} \times\left(\mathrm{SL}_{2} \times \mathrm{SL}_{3}\right)$ representation $V_{10} \otimes\left(V_{1} \otimes W_{2}\right)$ and identify it with the vector bundle $V_{10} \otimes \mathcal{E}$ over $G\left(11, V_{1} \otimes W_{2}\right)$, where $\mathcal{E}$ is the universal subbundle. In this case, we have

$$
G\left(11, V_{1} \otimes W_{2}\right)=\mathbb{P}\left(V_{1} \otimes W_{2}\right)^{\vee}=\mathbb{P}\left(V_{1}^{\vee} \otimes W_{2}^{\vee}\right) .
$$

By identifying $\mathrm{GL}_{n}=\mathrm{GL}\left(\mathbb{C}^{n}\right)$ with $\mathrm{GL}\left(\left(\mathbb{C}^{n}\right)^{\vee}\right)$ through the dual representation, we can apply the result of $\S 5.2$ to the $\mathrm{PGL}_{2} \times \mathrm{PGL}_{3}$-action on $\mathbb{P}\left(V_{1}^{\vee} \otimes W_{2}^{\vee}\right)$. Thus we find that it is almost transitive with stabilizer of a general point $[H] \in \mathbb{P}\left(V_{1} \otimes W_{2}\right)^{\vee}$ isomorphic to $\mathfrak{S}_{4}$, and the corresponding hyperplane $H \subset \mathbb{P}\left(V_{1} \otimes W_{2}\right)$ isomorphic to

$$
\mathbb{P}\left(\epsilon \oplus \theta^{\oplus 2} \oplus \psi \oplus \epsilon \psi\right)^{\vee} \simeq \mathbb{P}\left(\epsilon \oplus \theta^{\oplus 2} \oplus \psi \oplus \epsilon \psi\right)
$$

as an $\mathfrak{\subseteq}_{4}$-space. We set $V=\epsilon \oplus \theta^{\oplus 2} \oplus \psi \oplus \epsilon \psi$.

We apply the slice method to the projection $\mathbb{P}\left(V_{10} \otimes \mathcal{E}\right) \rightarrow \mathbb{P}\left(V_{1} \otimes W_{2}\right)^{\vee}$. This gives

$$
\mathbb{P}\left(V_{10} \otimes \mathcal{E}\right) / \mathrm{PGL}_{2} \times \mathrm{PGL}_{2} \times \mathrm{PGL}_{3} \sim \mathbb{P}\left(V_{10} \otimes V\right) / \mathrm{PGL}_{2} \times \Xi_{4} .
$$




\section{RATIONALITY OF SOME TETRAGONAL LOCI}

Next we use the no-name method for the projection $\mathbb{P}\left(V_{10} \otimes V\right) \rightarrow \mathbb{P}\left(V_{10} \otimes \psi\right)$ from the rest summand $V_{10} \otimes\left(\epsilon \oplus \theta^{\oplus 2} \oplus \epsilon \psi\right)$. Then we have

$$
\mathbb{P}\left(V_{10} \otimes V\right) / \mathrm{PGL}_{2} \times \mathbb{\Xi}_{4} \sim \mathbb{C}^{88} \times\left(\mathbb{P}\left(V_{10} \otimes \psi\right) / \mathrm{PGL}_{2} \times \mathfrak{\Xi}_{4}\right) .
$$

Finally, we apply Lemma 4.4 to the $\mathrm{PGL}_{2} \times \Xi_{4}$-representation $V_{10} \otimes \psi$. The group $\mathrm{PGL}_{2}$ acts on $G\left(3, V_{10}\right)$ almost freely by Lemma 4.3 . Therefore the quotient $G\left(3, V_{10}\right) / \mathrm{SL}_{2}$ is stably rational of level two by Lemma 4.1 (1) and the rationality of $\mathbb{P} V_{10} / \mathrm{SL}_{2}$ ([BK85]). On the other hand, $\mathbb{P}\left(\psi^{\oplus 3}\right) / \mathfrak{S}_{4}$ is rational by Proposition 5.4 . Hence by Lemma 4.4 we conclude that $\mathbb{P}\left(V_{10} \otimes \psi\right) / \mathrm{PGL}_{2} \times \mathfrak{S}_{4}$ is rational. This finishes the proof of Proposition 5.5 for $b=10$.

\section{The case of the blown-up $\mathbb{P}^{3}$}

In this section we study the cases $g \equiv 1,10$ (12) in Theorem 1.1 , where the basic $\mathbb{P}^{2}$-bundle is $X_{0,1}$, the blow-up of $\mathbb{P}^{3}$ along a line $l$. We keep the notation of $\S 2.2$.

\subsection{The case $g \equiv 10(12)$}

Let $b>0$ be an odd number. Let $\mathcal{L} \rightarrow\left|L_{2, b}\right|$ be the tautological bundle and let $\mathcal{E} \rightarrow\left|L_{2, b}\right|$ be the bundle $H^{0}\left(L_{2, b+1}\right) / \mathcal{L} \otimes H^{0}\left(L_{0,1}\right)$ as defined in Proposition 3.1. Since $L_{2, b+1}$ is $\operatorname{Aut}\left(X_{0,1}\right)$ linearized by Lemma 2.3 , the bundle $\mathcal{E}$ is $\operatorname{Aut}\left(X_{0,1}\right)$-linearized. The quotient $\mathbb{P} \mathcal{E} / \operatorname{Aut}\left(X_{0,1}\right)$ is birational to the tetragonal locus of genus $6 b+4 \equiv 10$ (12) by Proposition 3.1.

Lemma 6.1. The group $\operatorname{Aut}\left(X_{0,1}\right)$ acts on $\left|L_{2, b}\right|$ almost freely.

Proof. As in Lemma 5.1, a general member of $\left|L_{2, b}\right|$ is the blow-up of $\mathbb{P}^{2}$ at $3 b+3$ general points (put $s=3 b+2, n=s-1$ in [dCG93, $\S 2]$ ), and such a surface has no nontrivial automorphism; see [Koi88] for the case $b \geqslant 2$, while the case $b=1$ is well known. (See also Lemma 6.3 for another approach.)

Proposition 6.2. The quotient $\mathbb{P} \mathcal{E} / \operatorname{Aut}\left(X_{0,1}\right)$ is rational.

Proof. By the no-name lemma we see that

$$
\mathbb{P} \mathcal{E} / \operatorname{Aut}\left(X_{0,1}\right) \sim \mathbb{P}^{6 b+13} \times\left(\left|L_{2, b}\right| / \operatorname{Aut}\left(X_{0,1}\right)\right),
$$

so it suffices to show that $\left|L_{2, b}\right| / \operatorname{Aut}\left(X_{0,1}\right)$ is stably rational of level $6 b+13$. Consider the product $U=\left|L_{2, b}\right| \times\left|L_{2,1}\right|$. We can identify the first projection $U \rightarrow\left|L_{2, b}\right|$ with the projective bundle $\mathbb{P}\left(\mathcal{L} \otimes H^{0}\left(L_{2,1}\right)\right)$, and the second $U \rightarrow\left|L_{2,1}\right|$ with $\mathbb{P}\left(\mathcal{L}^{\prime} \otimes H^{0}\left(L_{2, b}\right)\right)$, where $\mathcal{L}^{\prime} \rightarrow\left|L_{2,1}\right|$ is the tautological bundle. Since $L_{4, b+1}$ is $\operatorname{Aut}\left(X_{0,1}\right)$-linearized by Lemma 2.3, both $\mathcal{L} \otimes H^{0}\left(L_{2,1}\right)$ and $\mathcal{L}^{\prime} \otimes H^{0}\left(L_{2, b}\right)$ are $\operatorname{Aut}\left(X_{0,1}\right)$-linearized. By the no-name method, we obtain

$$
\begin{aligned}
U / \operatorname{Aut}\left(X_{0,1}\right) & \sim \mathbb{P}^{15} \times\left(\left|L_{2, b}\right| / \operatorname{Aut}\left(X_{0,1}\right)\right) \\
& \sim \mathbb{P}^{6 b+9} \times\left(\left|L_{2,1}\right| / \operatorname{Aut}\left(X_{0,1}\right)\right) .
\end{aligned}
$$

We shall prove that $\left|L_{2,1}\right| / \operatorname{Aut}\left(X_{0,1}\right)$ is stably rational of level one. Recall that $\left|L_{2,1}\right|$ is identified with the linear system of cubic surfaces in $\mathbb{P}^{3}$ containing the line $l$. Thus, if we consider the parameter space

$$
V=\left\{\left(S, l^{\prime}\right) \in\left|\mathcal{O}_{\mathbb{P}^{3}}(3)\right| \times \mathbb{G}\left(1, \mathbb{P}^{3}\right) \mid l^{\prime} \subset S\right\},
$$

then $\left|L_{2,1}\right| / \operatorname{Aut}\left(X_{0,1}\right)$ is birational to $V / \mathrm{PGL}_{4}$, the moduli space of cubic surfaces with a line on it. Let $\mathcal{F} \rightarrow V$ be the pullback of the universal subbundle over $\mathbb{G}\left(1, \mathbb{P}^{3}\right)$. We have

$$
\mathbb{P} \mathcal{F}=\left\{\left(S, l^{\prime}, p\right) \in\left|\mathcal{O}_{\mathbb{P}^{3}}(3)\right| \times \mathbb{G}\left(1, \mathbb{P}^{3}\right) \times \mathbb{P}^{3} \mid p \in l^{\prime} \subset S\right\} .
$$




\section{SHOUHEI MA}

Let $\mathcal{F}^{\prime}$ be the twist of $\mathcal{F}$ by the pullback of $\mathcal{O}_{\left|\mathcal{O}_{\mathbb{P} 3}(3)\right|}(1)$. We can identify $\mathbb{P} \mathcal{F}$ with $\mathbb{P} \mathcal{F}^{\prime}$, and $\mathcal{F}^{\prime}$ is $\mathrm{PGL}_{4}$-linearized because $\sqrt{-1} \in \mathrm{SL}_{4}$ acts on it trivially. By the no-name lemma for $\mathcal{F}^{\prime}$ we have

$$
\mathbb{P} \mathcal{F} / \mathrm{PGL}_{4} \sim \mathbb{P}^{1} \times\left(V / \mathrm{PGL}_{4}\right) .
$$

On the other hand, consider the space $T$ of flags $p \in l^{\prime} \subset H \subset \mathbb{P}^{3}$, where $H$ is a plane. We have the $\mathrm{PGL}_{4}$-equivariant map

$$
\mathbb{P} \mathcal{F} \rightarrow T, \quad\left(S, l^{\prime}, p\right) \mapsto\left(p \in l^{\prime} \subset T_{p} S\right) .
$$

Its fiber over $\left(p \in l^{\prime} \subset H\right) \in T$ is an open subset of a linear system $\mathbb{P} W$ in $\left|\mathcal{O}_{\mathbb{P} 3}(3)\right|$. The group $\mathrm{GL}_{4}$ acts on $T$ transitively; the stabilizer $G$ of $\left(p \in l^{\prime} \subset H\right)$ is connected and solvable. By the slice method for (6.1) we see that

$$
\mathbb{P} \mathcal{F} / \mathrm{PGL}_{4} \sim \mathbb{P} W / G,
$$

and $\mathbb{P} W / G$ is rational by Miyata's theorem [Miy71]. Hence $\left|L_{2,1}\right| / \operatorname{Aut}\left(X_{0,1}\right)$ is stably rational of level one. This finishes the proof of Proposition 6.2.

6.2 The case $g \equiv 1(12)$

Let $b>0$ be an even number. Then $L_{2, b}$ is $\operatorname{Aut}\left(X_{0,1}\right)$-linearized by Lemma 2.3. The quotient $\mathbb{G}\left(1,\left|L_{2, b}\right|\right) / \operatorname{Aut}\left(X_{0,1}\right)$ is birational to the tetragonal locus of genus $6 b+1 \equiv 1(12)$.

Before proving its rationality, we recall that the $\operatorname{Aut}\left(X_{0,1}\right)$-representation $H^{0}\left(L_{2, b}\right)$ is reducible: it has the invariant filtration

$$
0 \subset H^{0}\left(L_{2,-2}\right) \otimes H^{0}\left(L_{0, b+2}\right) \subset H^{0}\left(L_{1,-1}\right) \otimes H^{0}\left(L_{1, b+1}\right) \subset H^{0}\left(L_{2, b}\right)
$$

defined by the vanishing orders along $\Sigma$. Here $H^{0}\left(L_{d,-d}\right)$ is one-dimensional and defines $d \Sigma$. If we consider $H^{0}\left(L_{2, b}\right)$ as a representation of the double cover

$$
\tilde{G}=\mathrm{SL}_{2} \ltimes \operatorname{Hom}\left(V_{1}, V_{1}\right) \rtimes \mathrm{GL}_{2}
$$

of $\operatorname{Aut}\left(X_{0,1}\right)$, the successive quotients of (6.2) are the $\mathrm{SL}_{2} \times \mathrm{GL}_{2}$-representations

$$
V_{b+2,0}, \quad V_{b+1,1}, \quad V_{b, 2},
$$

with the action of $\operatorname{Hom}\left(V_{1}, V_{1}\right)$ as described in (2.4). This structure of $H^{0}\left(L_{2, b}\right)$ was first observed in the case $b=1$ by Böhning-Bothmer-Casnati [BvBC12].

We consider the quotient representation

$$
W=H^{0}\left(L_{2, b}\right) /\left(H^{0}\left(L_{2,-2}\right) \otimes H^{0}\left(L_{0, b+2}\right)\right) .
$$

Geometrically the quotient map $H^{0}\left(L_{2, b}\right) \rightarrow W$ gives the Taylor expansions up to first order of the sections of $L_{2, b}$ along $\Sigma$.

Lemma 6.3. The group $\operatorname{Aut}\left(X_{0,1}\right)$ acts on $\mathbb{P} W=\mathbb{P}\left(V_{b+1,1} \oplus V_{b, 2}\right)$ almost freely.

Proof. Suppose that for a general point $\left[F_{1}, F_{2}\right] \in \mathbb{P}\left(V_{b+1,1} \oplus V_{b, 2}\right)$ we have an element $\left(g_{1}, h, g_{2}\right) \in \tilde{G}$ fixing it. Consider the projection $\mathbb{P} W \rightarrow \mathbb{P} V_{b, 2}$ from $V_{b+1,1}$ which is $\tilde{G}$-equivariant. Since PGL $\mathrm{PG}_{2} \times \mathrm{PGL}_{2}$ acts on $\mathbb{P} V_{b, 2}$ almost freely, we must have $\left(g_{1}, g_{2}\right)=( \pm 1, \lambda)$ for some scalar $\lambda \in \mathbb{C}^{\times}$. Composing it with $(-1,-1) \in \mathrm{SL}_{2} \times \mathrm{GL}_{2}$, we may assume that $g_{1}=1$. Now $(1, h, \lambda)$ maps $\left[F_{1}, F_{2}\right]$ to $\left[\lambda^{-1} F_{1}+\lambda^{-1}\left\langle F_{2}, h\right\rangle, F_{2}\right]$, where $\left\langle F_{2}, \cdot\right\rangle: \operatorname{Hom}\left(V_{1}, V_{1}\right) \rightarrow V_{b+1,1}$ is the linear map induced by the multiplication and the contraction. Thus we have $(\lambda-1) F_{1}=\left\langle F_{2}, h\right\rangle$. Since the map $\left\langle F_{2}, \cdot\right\rangle$ is injective for general $F_{2} \in V_{b, 2}$, choosing $F_{1}$ generically we have $\lambda=1$ and $h=0$.

Now we prove the following result. 


\section{RATIONALITY OF SOME TETRAGONAL LOCI}

Proposition 6.4. The quotient $\mathbb{G}\left(1,\left|L_{2, b}\right|\right) / \operatorname{Aut}\left(X_{0,1}\right)$ is rational.

Proof. In the first step, we apply Lemma 4.2 to the quotient map $H^{0}\left(L_{2, b}\right) \rightarrow W$. By Lemma 6.3 and Lemma 4.3 , Aut $\left(X_{0,1}\right)$ acts on $\mathbb{G}(1, \mathbb{P} W)$ almost freely. Then we obtain

$$
\mathbb{G}\left(1,\left|L_{2, b}\right|\right) / \operatorname{Aut}\left(X_{0,1}\right) \sim \mathbb{C}^{2 b+6} \times\left(\mathbb{G}(1, \mathbb{P} W) / \operatorname{Aut}\left(X_{0,1}\right)\right) .
$$

By Lemma 4.1 (1) we have

$$
\mathbb{P}^{1} \times\left(\mathbb{G}(1, \mathbb{P} W) / \operatorname{Aut}\left(X_{0,1}\right)\right) \sim \mathbb{P}^{5 b+5} \times\left(\mathbb{P} W / \operatorname{Aut}\left(X_{0,1}\right)\right) .
$$

We shall use the no-name method for $\mathbb{P} W \times\left(\left|L_{2,0}\right| \times\left|L_{1,1}\right|\right)$. Since both $L_{2,0}$ and $L_{1,1}$ are Aut $\left(X_{0,1}\right)$ linearized and since $\operatorname{Aut}\left(X_{0,1}\right)$ acts on $\left|L_{2,0}\right| \times\left|L_{1,1}\right|$ almost freely, we see that

$$
\begin{aligned}
\left(\mathbb{P} W \times\left|L_{2,0}\right| \times\left|L_{1,1}\right|\right) / \operatorname{Aut}\left(X_{0,1}\right) & \sim \mathbb{P}^{9} \times \mathbb{P}^{6} \times\left(\mathbb{P} W / \operatorname{Aut}\left(X_{0,1}\right)\right) \\
& \sim \mathbb{P}^{5 b+6} \times\left(\left(\left|L_{2,0}\right| \times\left|L_{1,1}\right|\right) / \operatorname{Aut}\left(X_{0,1}\right)\right) .
\end{aligned}
$$

In this way, the problem is reduced to showing that $\left(\left|L_{2,0}\right| \times\left|L_{1,1}\right|\right) / \operatorname{Aut}\left(X_{0,1}\right)$ is stably rational of level $5 b+6$. Actually, we shall prove that it is rational.

We identify $\operatorname{Aut}\left(X_{0,1}\right)$ with the stabilizer in $\mathrm{PGL}_{4}$ of the line $l,\left|L_{2,0}\right|$ with $\left|\mathcal{O}_{\mathbb{P}^{3}}(2)\right|$, and $\left|L_{1,1}\right|$ with the linear system of quadrics containing $l$. This implies that $\operatorname{Aut}\left(X_{0,1}\right)$ acts on $\left|L_{1,1}\right|$ almost transitively, with the stabilizer of a general $Q \in\left|L_{1,1}\right|$ isomorphic to $\left(\mathbb{C}^{\times} \ltimes \mathbb{C}\right) \times \mathrm{PGL}_{2}$ (which is the stabilizer of $l$ in $\operatorname{Aut}(Q))$. By the slice method for the projection $\left|L_{2,0}\right| \times\left|L_{1,1}\right| \rightarrow\left|L_{1,1}\right|$ we obtain

$$
\begin{aligned}
\left(\left|L_{2,0}\right| \times\left|L_{1,1}\right|\right) / \operatorname{Aut}\left(X_{0,1}\right) & \sim\left|L_{2,0}\right| /\left(\mathbb{C}^{\times} \ltimes \mathbb{C}\right) \times \mathrm{PGL}_{2} \\
& \sim \mathbb{C} \times\left(\left|\mathcal{O}_{Q}(2,2)\right| /\left(\mathbb{C}^{\times} \ltimes \mathbb{C}\right) \times \mathrm{PGL}_{2}\right) .
\end{aligned}
$$

Consider the product $U=\left|\mathcal{O}_{Q}(1,0)\right| \times\left|\mathcal{O}_{Q}(2,2)\right|$. Note that $\mathcal{O}_{Q}(1,0)$ and $\mathcal{O}_{Q}(2,2)$ are both $\left(\mathbb{C}^{\times} \ltimes \mathbb{C}\right) \times \mathrm{PGL}_{2}$-linearized. By the no-name lemma for the second projection $U \rightarrow\left|\mathcal{O}_{Q}(2,2)\right|$, we have

$$
U /\left(\mathbb{C}^{\times} \ltimes \mathbb{C}\right) \times \mathrm{PGL}_{2} \sim \mathbb{P}^{1} \times\left(\left|\mathcal{O}_{Q}(2,2)\right| /\left(\mathbb{C}^{\times} \ltimes \mathbb{C}\right) \times \mathrm{PGL}_{2}\right) .
$$

On the other hand, using the slice method for the first projection $U \rightarrow\left|\mathcal{O}_{Q}(1,0)\right|$, we deduce that

$$
U /\left(\mathbb{C}^{\times} \ltimes \mathbb{C}\right) \times \mathrm{PGL}_{2} \sim\left|\mathcal{O}_{Q}(2,2)\right| / \mathbb{C}^{\times} \times \mathrm{PGL}_{2} .
$$

The last quotient is rational by Katsylo [Kat84b]. Thus $\left(\left|L_{2,0}\right| \times\left|L_{1,1}\right|\right) / \operatorname{Aut}\left(X_{0,1}\right)$ is rational, and the proof of the proposition is completed.

\section{The case of the small resolution of a quadric cone}

In this section we study the cases $g \equiv 2,5$ (12) in Theorem 1.1 , where the basic $\mathbb{P}^{2}$-bundle is $X_{1,1}$, a small resolution of a quadric cone. We use the notation of $\S 2.3$ freely.

7.1 The case $g \equiv 2(12)$

Let $b>0$ be an odd number. Let $\mathcal{L} \rightarrow\left|L_{2, b}\right|$ be the tautological bundle and let $\mathcal{E} \rightarrow\left|L_{2, b}\right|$ be the bundle $H^{0}\left(L_{2, b+1}\right) / \mathcal{L} \otimes H^{0}\left(L_{0,1}\right)$ as defined in Proposition 3.1. Then $\mathbb{P} \mathcal{E} / \operatorname{Aut}\left(X_{1,1}\right)$ is birational to the tetragonal locus of genus $6 b+8 \equiv 2(12)$. Note that $\mathcal{E}$ is $\operatorname{Aut}\left(X_{1,1}\right)$-linearized because $L_{2, b+1}$ is so by Lemma 2.5.

Lemma 7.1. The group $\operatorname{Aut}\left(X_{1,1}\right)$ acts on $\left|L_{2, b}\right|$ almost freely. 


\section{SHOUHEI MA}

Proof. As in Lemma 5.1, a general member $S$ of $\left|L_{2, b}\right|$ is the blow-up of $\mathbb{P}^{2}$ at $3 b+5$ general points (put $s=3 b+4, n=s-1$ in [dCG93, $\S 2]$ ). When $b \geqslant 2, S$ has no nontrivial automorphism ([Koi88]). On the other hand, when $b=1, S$ has the Geiser involution but this does not preserve the line bundle giving the embedding $S \subset X_{1,1}$.

Proposition 7.2. The quotient $\mathbb{P} \mathcal{E} / \operatorname{Aut}\left(X_{1,1}\right)$ is rational.

Proof. By the no-name lemma we have

$$
\mathbb{P} \mathcal{E} / \operatorname{Aut}\left(X_{1,1}\right) \sim \mathbb{P}^{6 b+17} \times\left(\left|L_{2, b}\right| / \operatorname{Aut}\left(X_{1,1}\right)\right) .
$$

To deduce the stable rationality of $\left|L_{2, b}\right| / \operatorname{Aut}\left(X_{1,1}\right)$, we consider the product $U=\left|L_{2, b}\right| \times$ $\left(\left|L_{0,1}\right| \times\left|L_{1,-1}\right|\right)$. We can identify the projection $\left|L_{2, b}\right| \times\left|L_{0,1}\right| \rightarrow\left|L_{2, b}\right|$ with the projective bundle $\mathbb{P}\left(\mathcal{L} \otimes H^{0}\left(L_{0,1}\right)\right)$, and $\left|L_{2, b}\right| \times\left|L_{1,-1}\right| \rightarrow\left|L_{2, b}\right|$ with $\mathbb{P}\left(\mathcal{L} \otimes H^{0}\left(L_{1,-1}\right)\right)$. Note that $\mathcal{L} \otimes H^{0}\left(L_{0,1}\right)$ and $\mathcal{L} \otimes H^{0}\left(L_{1,-1}\right)$ are $\operatorname{Aut}\left(X_{1,1}\right)$-linearized by Lemma 2.5. By the no-name lemma we obtain

$$
U / \operatorname{Aut}\left(X_{1,1}\right) \sim \mathbb{P}^{1} \times \mathbb{P}^{1} \times\left(\left|L_{2, b}\right| / \operatorname{Aut}\left(X_{1,1}\right)\right) .
$$

On the other hand, we use the slice method for the projection $U \rightarrow\left|L_{0,1}\right| \times\left|L_{1,-1}\right|$. Since $\left|L_{0,1}\right| \times\left|L_{1,-1}\right|$ is identified with the base quadric $Q_{0}$ of the quadric cone, we see that $\left(\mathrm{SL}_{2} \times\right.$ $\left.\mathrm{GL}_{2}\right) \ltimes V_{1,1}$ acts on it transitively. If $G_{1}$ is the stabilizer in $\mathrm{SL}_{2} \times \mathrm{GL}_{2}$ of a point $p$ of $Q_{0}$, then we have

$$
U / \operatorname{Aut}\left(X_{1,1}\right) \sim\left|L_{2, b}\right| / G_{1} \ltimes V_{1,1} .
$$

Since $G_{1}$ is connected and solvable, so is $G_{1} \ltimes V_{1,1}$. Thus $\left|L_{2, b}\right| / G_{1} \ltimes V_{1,1}$ is rational by Miyata's theorem [Miy71]. This shows that $\left|L_{2, b}\right| / \operatorname{Aut}\left(X_{1,1}\right)$ is stably rational of level two, and the proposition is proved.

7.2 The case $g \equiv 5(12)$

Let $b>0$ be an even number. By Proposition 3.1, the quotient $\mathbb{G}\left(1,\left|L_{2, b}\right|\right) / \operatorname{Aut}\left(X_{1,1}\right)$ is birational to the tetragonal locus of genus $6 b+5 \equiv 5(12)$. Here $L_{2, b}$ is $\operatorname{Aut}\left(X_{1,1}\right)$-linearized by Lemma 2.5.

Let $F$ be the kernel of the restriction map $H^{0}\left(L_{2, b}\right) \rightarrow H^{0}\left(\mathcal{O}_{\sigma}(b)\right)$. Recall that the $\operatorname{Aut}\left(X_{1,1}\right)$ representation $H^{0}\left(L_{2, b}\right)$ is reducible, having the invariant filtration

$$
0 \subset f^{*} H^{0}\left(\mathcal{O}_{Q_{0}}(b+2,2)\right) \subset F \subset H^{0}\left(L_{2, b}\right)
$$

defined by the vanishing orders along $\sigma$. If we consider $H^{0}\left(L_{2, b}\right)$ as a representation of the double cover $\left(\mathrm{SL}_{2} \times \mathrm{GL}_{2}\right) \ltimes V_{1,1}$ of $\operatorname{Aut}\left(X_{1,1}\right)$, the successive quotients of (7.1) are the $\mathrm{SL}_{2} \times \mathrm{GL}_{2^{-}}$ representations

$$
V_{b+2,2}, \quad V_{b+1,1}, \quad V_{b, 0},
$$

and the unipotent radical $V_{1,1} \ni h$ acts by multiplication by $\exp (h)$,

$$
V_{b+1,1} \rightarrow V_{b+1,1} \oplus V_{b+2,2}, \quad V_{b, 0} \rightarrow V_{b, 0} \oplus V_{b+1,1} \oplus V_{b+2,2} .
$$

We consider the quotient representation

$$
W=H^{0}\left(L_{2, b}\right) / f^{*} H^{0}\left(\mathcal{O}_{Q_{0}}(b+2,2)\right) .
$$

Let $\left(\left[X_{0}, X_{1}\right],\left[Y_{0}, Y_{1}\right]\right)$ be bi-homogeneous coordinates of $Q_{0} \simeq \mathbb{P}^{1} \times \mathbb{P}^{1}$.

LEMma 7.3. The following hold.

(1) When $b \geqslant 4$, Aut $\left(X_{1,1}\right)$ acts on $\mathbb{P} W$ and $\mathbb{G}(1, \mathbb{P} W)$ almost freely.

(2) When $b=2$, Aut $\left(X_{1,1}\right)$ acts on $\mathbb{G}(1, \mathbb{P} W)$ almost freely. 


\section{RATIONALITY OF SOME TETRAGONAL LOCI}

(3) When $b=2$, Aut $\left(X_{1,1}\right)$ acts on $\mathbb{P} W=\mathbb{P}\left(V_{3,1} \oplus V_{2,0}\right)$ almost transitively.

If $v=\left(X_{0}^{3} Y_{1}+X_{1}^{3} Y_{0}, X_{0} X_{1}\right) \in W$, the stabilizer of $[v] \in \mathbb{P} W$ is the subgroup

$$
\mathfrak{S}_{2} \ltimes \mathbb{C}^{\times} \subset \mathrm{SL}_{2} \times \mathrm{GL}_{2} /(-1,-1)
$$

generated by

$$
\begin{gathered}
\mathfrak{\Xi}_{2}: X_{0} \mapsto X_{1}, \quad X_{1} \mapsto-X_{0}, \quad Y_{0} \mapsto Y_{1}, \quad Y_{1} \mapsto-Y_{0}, \\
\alpha \in \mathbb{C}^{\times}: X_{0} \mapsto \alpha X_{0}, \quad X_{1} \mapsto \alpha^{-1} X_{1}, \quad Y_{0} \mapsto \alpha^{3} Y_{0}, \quad Y_{1} \mapsto \alpha^{-3} Y_{1} .
\end{gathered}
$$

Proof. (1) In view of Lemma 4.3, we prove the assertion only for $\mathbb{P} W=\mathbb{P}\left(V_{b+1,1} \oplus V_{b, 0}\right)$. Let $K \subset$ $\mathrm{SL}_{2}$ be the stabilizer of a general point $[F] \in \mathbb{P} V_{b, 0}$. It suffices to show that $\left(K \times \mathrm{GL}_{2}\right) \ltimes V_{1,1}$ modulo $(-1,-1)$ acts on $(\mathbb{C} F)^{\vee} \otimes V_{b+1,1}$ almost freely. Here $V_{1,1}$ acts as translation by $F^{\vee} \otimes\left(V_{1,1} \cdot F\right)$. Consider the quotient map

$$
(\mathbb{C} F)^{\vee} \otimes V_{b+1,1} \rightarrow(\mathbb{C} F)^{\vee} \otimes V_{b+1,1} /\left((\mathbb{C} F)^{\vee} \otimes\left(V_{1,1} \cdot F\right)\right) .
$$

This is a $K \times \mathrm{GL}_{2}$-linearized vector bundle on which $V_{1,1}$ acts by translations in the fibers (in particular, freely). If we set $U=(\mathbb{C} F)^{\vee} \otimes\left(V_{b+1} / V_{1} \cdot F\right)$, the image of (7.3) is the $K \times \mathrm{GL}_{2^{-}}$ representation $U \otimes V_{1}$. It is easy to see that $K \times \mathrm{GL}_{2} /(-1,-1)$ acts on $U \otimes V_{1}$ almost freely. When $b \geqslant 6$, we have $K=\{ \pm 1\}$ and $\mathrm{GL}_{2}$ acts on $V_{1}^{\oplus b}$ almost freely. When $b=4$, the quotient $K / \pm 1$ is the Klein four-group which acts on $G(2, U)$ effectively. Then our assertion follows by considering the fibration $U \otimes V_{1} \rightarrow G(2, U)$ as in (4.3).

(2) A general two-dimensional linear subspace of $W$ can be normalized by the $\operatorname{Aut}\left(X_{1,1}\right)$-action to the following type:

$$
P=\left\langle\left(F_{0}, X_{0}^{2}\right),\left(F_{1}, X_{1}^{2}\right)\right\rangle, \quad F_{i} \in V_{3,1} .
$$

The basis presented here is canonical, in that its image by the projection $\pi: W \rightarrow V_{2,0}$ gives the discriminant locus of the conic pencil $\pi(P)$. Hence any stabilizer of $P$ preserves this basis up to scalar multiplication. Using this property, our assertion follows from a direct calculation.

(3) We only have to determine the stabilizer. Clearly the group $\mathfrak{S}_{2} \ltimes \mathbb{C}^{\times}$defined above fixes $[v]$. Conversely, suppose $g \in \operatorname{Aut}\left(X_{1,1}\right)$ fixes $[v]$. Composing $g$ with an element of $\mathfrak{\Xi}_{2} \ltimes \mathbb{C}^{\times}$, we may assume that $g$ is the projection image of an element of the form $\left(1, g_{2}, h\right) \in\left(\mathrm{SL}_{2} \times \mathrm{GL}_{2}\right) \ltimes V_{1,1}$. Then we would have

$$
X_{0}^{3}\left(g_{2}\left(Y_{1}\right)-Y_{1}\right)+X_{1}^{3}\left(g_{2}\left(Y_{0}\right)-Y_{0}\right)=-h X_{0} X_{1},
$$

from which it follows that $h=0$ and $g_{2}=1$.

Now we prove the following result.

Proposition 7.4. The quotient $\mathbb{G}\left(1,\left|L_{2, b}\right|\right) / \operatorname{Aut}\left(X_{1,1}\right)$ is rational.

Proof. By Lemma 7.3 (1) and (2), we can apply Lemma 4.2 to the quotient homomorphism $H^{0}\left(L_{2, b}\right) \rightarrow W$. Then we obtain

$$
\mathbb{G}\left(1,\left|L_{2, b}\right|\right) / \operatorname{Aut}\left(X_{1,1}\right) \sim \mathbb{C}^{6 b+18} \times\left(\mathbb{G}(1, \mathbb{P} W) / \operatorname{Aut}\left(X_{1,1}\right)\right) .
$$

When $b \geqslant 4$, we can use Lemma 4.1 (1) to see that

$$
\mathbb{P}^{1} \times\left(\mathbb{G}(1, \mathbb{P} W) / \operatorname{Aut}\left(X_{1,1}\right)\right) \sim \mathbb{P}^{3 b+3} \times\left(\mathbb{P} W / \operatorname{Aut}\left(X_{1,1}\right)\right),
$$




\section{SHOUHEI MA}

so the problem is reduced to proving the stable rationality of $\mathbb{P} W / \operatorname{Aut}\left(X_{1,1}\right)$ of level $3 b+3$. We shall use the duality $\operatorname{Aut}\left(X_{1,1}\right) \simeq \operatorname{Aut}\left(X_{0,1}\right)$ from Lemma 2.1. In the proof of Proposition 6.4, we have found representations $U_{1}$ and $U_{2}$ of $\operatorname{Aut}\left(X_{0,1}\right)$ of dimension 10 and 7 , respectively, such that $\operatorname{Aut}\left(X_{0,1}\right)$ acts on $\mathbb{P} U_{1} \times \mathbb{P} U_{2}$ almost freely with rational quotient. Replacing $\operatorname{Aut}\left(X_{0,1}\right)$ with $\operatorname{Aut}\left(X_{1,1}\right)$, we can repeat the same no-name argument for $\mathbb{P} W \times\left(\mathbb{P} U_{1} \times \mathbb{P} U_{2}\right)$ to deduce the stable rationality of $\mathbb{P} W / \operatorname{Aut}\left(X_{1,1}\right)$ of level 15 . This proves our assertion for $b \geqslant 4$.

Next we consider the case $b=2$. Let $v \in W$ be the vector as defined in Lemma $7.3(3)$. By Lemma 7.3 (2), (3) and Lemma 4.1 (2), we have

$$
\mathbb{P}^{1} \times\left(\mathbb{G}(1, \mathbb{P} W) / \operatorname{Aut}\left(X_{1,1}\right)\right) \sim \mathbb{P}(W / \mathbb{C} v) / \widetilde{\Xi}_{2} \ltimes \mathbb{C}^{\times},
$$

where $\mathfrak{S}_{2} \ltimes \mathbb{C}^{\times}$is as defined in Lemma 7.3 (3). It is easy to see the following $\mathfrak{S}_{2} \ltimes \mathbb{C}^{\times}$-decomposition of $W$ :

$$
\begin{gathered}
V_{2,0}=\left\langle X_{0} X_{1}\right\rangle \oplus\left\langle X_{0}^{2}, X_{1}^{2}\right\rangle, \\
V_{3,1}=\left\langle X_{0}^{3} Y_{1}+X_{1}^{3} Y_{0}\right\rangle \oplus\left\langle X_{0}^{3} Y_{1}-X_{1}^{3} Y_{0}\right\rangle \oplus \\
\left\langle X_{0}^{3} Y_{0}, X_{1}^{3} Y_{1}\right\rangle \oplus\left\langle X_{0}^{2} X_{1} Y_{0},-X_{0} X_{1}^{2} Y_{1}\right\rangle \oplus\left\langle X_{0} X_{1}^{2} Y_{0}, X_{0}^{2} X_{1} Y_{1}\right\rangle .
\end{gathered}
$$

Let $W_{i}$ be the representation of $\mathfrak{\Xi}_{2} \ltimes \mathbb{C}^{\times}$induced by the weight $i$ scalar representation of $\mathbb{C}^{\times}$, let $V_{\sigma}$ be the sign representation of $\mathfrak{S}_{2}$ pulled back to $\mathfrak{S}_{2} \ltimes \mathbb{C}^{\times}$, and let $V_{0}$ be the trivial representation. By the above calculation we have the decomposition

$$
W / \mathbb{C} v \simeq V_{0} \oplus V_{\sigma} \oplus W_{1}^{\oplus 2} \oplus W_{2} \oplus W_{3} .
$$

Here notice that our $\mathbb{C}^{\times}$is defined as the quotient by -1 of the $\alpha$-torus $\mathbb{C}^{\times}$in $(7.2)$, and this division by -1 reduces the weights of $\mathbb{C}^{\times}$-representations by half. Now $\mathfrak{S}_{2} \ltimes \mathbb{C}^{\times}$acts on $\mathbb{P}\left(W_{1} \oplus W_{2}\right)$ almost freely, so that we can apply the no-name lemma to the projection $\mathbb{P}(W / \mathbb{C} v) \rightarrow \mathbb{P}\left(W_{1} \oplus W_{2}\right)$. This gives

$$
\mathbb{P}(W / \mathbb{C} v) / \Xi_{2} \ltimes \mathbb{C}^{\times} \sim \mathbb{C}^{6} \times\left(\mathbb{P}\left(W_{1} \oplus W_{2}\right) / \mathfrak{S}_{2} \ltimes \mathbb{C}^{\times}\right) .
$$

Since $\mathbb{P}\left(W_{1} \oplus W_{2}\right) / \mathfrak{S}_{2} \ltimes \mathbb{C}^{\times}$has dimension two, it is rational. Therefore $\mathbb{G}(1, \mathbb{P} W) / \operatorname{Aut}\left(X_{1,1}\right)$ is stably rational of level one, and Proposition 7.4 is proved for $b=2$.

\section{REFERENCES}

AC81 E. Arbarello and M. Cornalba, Footnotes to a paper of Beniamino Segre, Math. Ann. 256 (1981), no. 3, 341-362. http://dx.doi.org/10.1007/BF01679702

Böh09 C. Böhning, The rationality problem in invariant theory, Progress in Math., to appear, arXiv:0904.0899 (2009).

BvBC12 C. Böhning, H.-C. Graf von Bothmer and G. Casnati, Birational properties of some moduli spaces related to tetragonal curves of genus 7, Int. Math. Res. Notices 2012, no. 22, 5219-5245. http://dx.doi.org/10.1093/imrn/rnr230

BK85 F.A. Bogomolov and P.I. Katsylo, Rationality of some quotient varieties, Mat. Sb. (N.S.) 126(168) (1985), 584-589. http://dx.doi.org/10.1070/SM1986v054n02ABEH002986

CdC04 G. Casnati and A. Del Centina, The rationality of Weierstrass space of type $(4, g)$, Math. Proc. Camb. Phil. Soc. 136 (2004), 53-66. http://dx.doi.org/10.1017/S0305004103007059

dCG93 A. Del Centina and A. Gimigliano, Scrollar invariants and resolutions of certain d-gonal curves, Ann. Univ. Ferrara Sez. VII (N.S.) 39 (1993), 187-201.

Gei12 F. Geiss, The unirationality of Hurwitz spaces of 6-gonal curves of small genus, Doc. Math. 17 (2012), 627-640. 


\section{RATIONALITY OF SOME TETRAGONAL LOCI}

Kat84a P.I. Katsylo, Rationality of the moduli spaces of hyperelliptic curves, Izv. Akad. Nauk SSSR. 48 (1984), 705-710.

Kat84b P.I. Katsylo, Rationality of fields of invariants of reducible representations of $\mathrm{SL}_{2}$, Mosc. Univ. Math. Bull. 39 (1984), 80-83.

Koi88 M. Koitabashi, Automorphism groups of generic rational surfaces, J. Algebra 116 (1988), no. 1, 130-142.

Ma12 S. Ma, The rationality of the moduli spaces of trigonal curves, Int. Math. Res. Notices, to appear, arXiv:1207.0184 (2012).

Ma13a S. Ma, The rationality of the moduli spaces of trigonal curves of odd genus, J. Reine Angew. Math. 683 (2013), 181-187. http://dx.doi.org/10.1515/crelle-2012-0003

Ma13b S. Ma, Rationality of fields of invariants for some representations of $\mathrm{SL}_{2} \times \mathrm{SL}_{2}$, Compositio Math. 149 (2013), no. 7, 1225-1234. http://dx.doi.org/10.1112/S0010437X13007069

Miy71 T. Miyata, Invariants of certain groups. I, Nagoya Math. J. 41 (1971), 69-73.

Pet23 K. Petri, Über die invariante Darstellung algebraischer Funktionen einer Veränderlichen, Math. Ann. 88 (1923), no. 3-4, 242-289.

Sch86 F.-O. Schreyer, Syzygies of canonical curves and special linear series, Math. Ann. 275 (1986), $105-137$.

Ser77 J.-P. Serre, Linear representations of finite groups, GTM 42, Springer-Verlag, 1977.

She85 N.I. Shepherd-Barron, The rationality of certain spaces associated to trigonal curves, Algebraic geometry, Bowdoin, 1985, 165-171, Proc. Symp. Pure Math. 46, Part 1, Amer. Math. Soc., Providence, 1987.

Shouhei Ma ma@math.nagoya-u.ac.jp

Graduate School of Mathematics, Nagoya University, Nagoya 464-8601, Japan 